UUITP-05/12

\title{
AKSZ construction from reduction data
}

\author{
Francesco Bonechi ${ }^{a}$, Alejandro Cabrera ${ }^{b}$ and Maxim Zabzine ${ }^{c}$ \\ ${ }^{a}$ I.N.F.N., Sezione di Firenze, \\ Via G. Sansone 1, 50019 Sesto Fiorentino - Firenze, Italy \\ ${ }^{b}$ Department of Mathematics, University of Toronto, Room 6290, 40 St. George Street, \\ Toronto, Ontario, Canada M5S 2E4; \\ Current Address: Departamento de Matematica Aplicada, Instituto de Matematica, \\ Universidade Federal do Rio de Janeiro, CEP 21941-909, Rio de Janeiro - RJ, Brazil. \\ ${ }^{c}$ Department of Physics and Astronomy, Uppsala University, \\ Box 516, SE-751 20 Uppsala, Sweden
}

\begin{abstract}
We discuss a general procedure to encode the reduction of the target space geometry into AKSZ sigma models. This is done by considering the AKSZ construction with target the BFV model for constrained graded symplectic manifolds. We investigate the relation between this sigma model and the one with the reduced structure. We also discuss several examples in dimension two and three when the symmetries come from Lie group actions and systematically recover models already proposed in the literature.
\end{abstract}




\section{Contents}

1 Introduction $\quad 2$

2 BV manifolds and AKSZ construction

2.1 BV quantization . . . . . . . . . . . . . . . . . . . . 5

2.2 The AKSZ construction .................... . . . . . . . .

3 The $B F V_{n}$ manifold 9

3.1 The $B F V_{n}$ construction from reduction data . . . . . . . . . . . . 12

4 The AKSZ-BFV system $\quad 15$

4.1 BV-BFV manifolds . . . . . . . . . . . . . . . . . . . 16

4.2 General features . . . . . . . . . . . . . . . . . . . 19

5 Reduction by group actions for $n=1 \quad 22$

$5.1 \quad B F V_{1}$ for Poisson actions . . . . . . . . . . . . . . . . 22

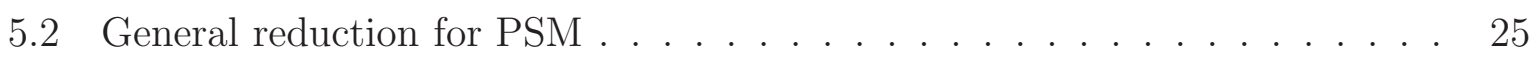

5.3 Reduction for zero modes of PSM . . . . . . . . . . . . . . 26

6 Reduction of a group action for $n=2 \quad 29$

6.1 Reduction of an exact Courant algebroid . . . . . . . . . . . . . 30

6.2 Courant algebroid related to a Poisson action . . . . . . . . . . 30

7 Summary and outlook

A Quantization of Hamiltonian systems with symmetry

B Basic facts of Poisson actions

C Reduction of Courant algebroids

\begin{tabular}{l|l|} 
D Relation to Poisson equivariant cohomology & 36 \\
\hline
\end{tabular}

E The zero modes case of AKSZ commuting with BFV 


\section{Introduction}

The idea of encoding the reduction of geometric structures into sigma models is rather old and proved to be very useful. The main motivation is to construct non trivial models with target $M / G$ by starting from the simpler one on $M$ and then gauging it. The geometrical reduction is then encoded in the gauge fixing of the new gauge degrees of freedom.

In a series of recent papers [35, 36] the procedure of gauging and reduction for the Poisson Sigma model (PSM in short) has been considered. The model is constructed when there is an action of a Lie group $G$ on a Poisson manifold $M$ by Poisson diffeomorphisms and this action is hamiltonian with momemntum map $\mu: M \rightarrow \mathfrak{g}^{*}$. It is important to note that this gauging procedure is not standard since it takes into account the topological nature of the PSM.

The main goal of this paper is to present a more general procedure which is analogous to gauging but adapted to general topological sigma models of AKSZ type as described below.

First, let us recall that the PSM is an example of the more general class of AKSZ (Aleksandrov-Kontsevich-Schwartz-Zaboronsky) topological field theories [1, 30]. For any graded symplectic finite dimensional manifold $\mathcal{M}$ of degree $n$, which comes equipped with an homological hamiltonian vector field, the AKSZ construction defines a topological sigma model in dimension $n+1$. The manifold $\mathcal{M}$ is called the target space and the hamiltonian on it can encode in a synthetic way various known geometrical structures. When the target manifold is non-negatively graded, the case $n=1$ corresponds to Poisson geometry and the corresponding model is the PSM; the case $n=2$ corresponds to Courant algebroid geometry and the model is called Courant sigma model (CSM).

Second, we recall that the graded manifold language offers a conceptually simple framework to deal with the reduction of these structures. Indeed, it becomes just ordinary symplectic reduction in which the reduced geometry is encoded in a reduced graded symplectic manifold $\mathcal{M}_{\text {red }}$ of the same type as the original $\mathcal{M}$. Poisson reduction as formulated for instance in [26] is easily reformulated in the langauge of hamiltonian reduction of $n=1$ symplectic graded manifolds [14]. The Courant algebroid reduction is the object of [9] and its formulation in the graded language can be found in [10].

Since one of the merits of the AKSZ method is the transparent relation between the sigma models and the underlying geometric structures, it seems reasonable that the machinery developed for reducing the latter can be encoded in the AKSZ formalism more efficiently and clearly than with the usual gauging procedures. In other words, it is natural 
to try to complete the following diagram

$$
\begin{array}{rll}
\text { geometric structures } & \stackrel{A K S Z}{\rightarrow} & \text { sigma models } \\
\downarrow \text { reduction } & \downarrow(?) \\
\text { geometric structures } & \stackrel{A K S Z}{\rightarrow} & \text { sigma models }
\end{array}
$$

In this paper we show that this can be done in a very general way by considering the so called BFV (Batalin-Fradkin-Vilkovisky) construction. This emerged in [2] as a hamiltonian version of BV-quantization and it later evolved into the pure symplectic geometry context (see [19, 20, 34]). In this paper, we shall use a variant of it which takes a graded symplectic manifold together with reduction data and produces another (higher dimensional) graded symplectic manifold $\mathcal{M}_{B F V}$ of the same type, which we shall refer to as BFV manifold. The BFV manifold encodes the reduction data in the homological vector field and the key point is to use $\mathcal{M}_{B F V}$ as a target for the AKSZ construction. This enlarged topological field theory is considered as the analogue of gauging the original sigma model.

Our main claim is that, by means of a formal argument, the AKSZ construction with target $\mathcal{M}_{B F V}$ can be shown to compute the correlators of the AKSZ theory corresponding to the reduced target $\mathcal{M}_{\text {red }}$. The argument is just formal since it needs to deal with integration over infinite dimensional spaces of fields. In turn, we provide exact arguments in the context of the underlying zero modes theory. We recall that the BV-space of zero modes is a finite dimensional theory which is a zero order approximation of the full AKSZ model (see [6, 7]) and which keeps the overall structure as in the general AKSZ construction. It can thus be taken as a partial verification of the full formal statement.

Finally, we present several examples in which the symmetries come from Lie group actions. We show how our construction yields, as particular cases, several gauged models already considered in the literature. We thus provide a more conceptual insight into these models and clarify how to produce new ones in which other types of reduced geometry can be also encoded.

Let us now discuss the content of the paper in more detail.

In Section 2 we review standard facts of $B V$ quantization and the AKSZ construction.

In Section 3 we introduce the notion of $B F V_{n}$ manifold. We choose an axiomatic approach rather than the usual construction which is postponed in Subsection 3.1. This construction is well known when applied to ungraded symplectic manifolds. Since we now apply it to graded manifolds, we can incorporate an underlying Q-hamiltonian into it. This results in the presence of non-standard terms in the so-called BFV charge and we thus provide details of its construction. 
In Section 4 we study the AKSZ space of fields when the target is a BFV manifold. In this case the space of fields inherits an additional grading and it is at the same time a $\mathrm{BFV}_{-1}$ and a BV manifold. This is what we define to be a $B V-B F V$-manifold and we show that it provides an homological model for BV-reduction. In subsection 4.1, we illustrate this by considering a toy model which starts with $\mathfrak{F}=T^{*}[-1] M$, where the finite dimensional smooth manifold $M$ is acted by a Lie group $G$. We show that the corresponding BV-BFV manifold $\mathfrak{F}_{B V-B F V}$ is defined by the choice of a volume form on the quotient $M / G$ and that the relevant integrals computed on $\mathfrak{F}_{B V-B F V}$ coincide with those on the quotient. We describe in Subsection 4.2 the general features of the AKSZ model with target a BFV manifold and we give a formal BV argument for the result shown in the finite dimensional example to be valid in general.

The rest of the paper is devoted to discuss explicit examples. In Section 5 we discuss the case of $n=1$, i.e. the encoding of Poisson reduction in the Poisson Sigma Model. We show that in the case of a Poisson action of Poisson-Lie group $G$, the $\mathrm{BFV}_{1}$ space is constructed by using the dual Poisson-Lie group $G^{*}$. Moreover, we extend the analysis of the general toy model to the finite dimensional spaces of zero modes. In Section 6 we discuss $\mathrm{BFV}_{2}$ spaces related to the reduction of exact Courant algebroids.

Finally, in Section 7 we present some conclusions and further directions. The Appendices are used to examine particular cases and give details of some arguments as indicated in the main text.

During the elaboration of this work, the paper [13] came out where a similar terminology is adopted. It is clear that it actually refers to a different construction. Indeed, in [13] the BV structure is defined on the space of fields on the bulk and the BFV on those on the boundary, while in our case they are defined on the same space of fields (see also example 4).

Notations and conventions. In the literature there exist inequivalent definitions of a graded manifold. In this paper for a graded manifold $\mathcal{M}$ we always mean a supermanifold $\mathcal{M}$ endowed with a compatible $\mathbb{Z}$-grading, i.e. a coordinate atlas in which each local coordinate $x^{a}$ is assigned a $\mathbb{Z}$-degree $\operatorname{deg} x^{a}$ and the coordinate transformations respect this degree. Moreover we will require that the parity is deg mod 2. This grading is encoded by the Euler vector field, that in the local coordinates $\left\{x^{a}\right\}$ reads

$$
\epsilon=\sum_{a}\left(\operatorname{deg} x^{a}\right) x^{a} \frac{\partial}{\partial x^{a}} .
$$

We denote with $C^{k}(\mathcal{M})$ the global (polynomial) functions of degree $k$, i.e. $f \in C^{k}(\mathcal{M})$ if $\epsilon(f)=k f$, and $C(\mathcal{M})=\bigoplus_{k} C^{k}(\mathcal{M}) \subset C^{\infty}(\mathcal{M})$. A symplectic form $\omega$ of degree $n$ is a 
closed, non degenerate 2-form such that $L_{\epsilon} \omega=n \omega$. It induces the structure of $n$-Poisson algebra on $C(\mathcal{M})$. When $n$ is odd, a generator for the Poisson bracket is a degree $-n$ linear map $\Delta: C(\mathcal{M}) \rightarrow C(\mathcal{M})$ satisfying

$$
\Delta(a b)=(\Delta a) b+(-)^{|a|} a(\Delta b)+(-)^{|a|}\{a, b\} \quad .
$$

We also require that $\Delta^{2}=0$ so that $\Delta$ becomes a derivation of the Poisson bracket. A $(-1)$-Poisson algebra together with a generator of the bracket will be called a $B V$-algebra.

Since in this paper there are a lot of different graded manifolds playing different roles, we try to help the reader by respecting the following notation: we use roman letters $M$ for ordinary smooth manifolds, capital $\mathcal{M}$ for graded manifolds and $\mathfrak{F}$ for $B V$ manifolds.

Acknowldegments. A.C. wants to thank several institutions for hospitality and support during the elaboration of this project: HIM institute (Bonn) where he participated on the Trimester Program on Geometry and Physics (2008) and both IMPA (Brazil) and the Math department of University of Toronto where he was a post-doctoral fellow. A.C. also thanks H.-C. Herbig for fruitful discussions on the BFV construction. F.B. wants to thank the authors of 10 for sharing their preliminary draft, the Department of Physics and Astronomy at Uppsala University and the Nordita for hospitality during the programs "Geometrical Aspects of String Theory" (2008) and "Geometry of Strings and Fields" (2011), when this project started and has been completed. The research of M.Z. is supported by VR grant 621-2011-5079.

\section{BV manifolds and AKSZ construction}

We review in this section the notion of BV manifold and the AKSZ construction of Topological Field Theories.

\subsection{BV quantization}

Definition $1 A B V$ manifold $\left(\mathfrak{F}, \omega_{-1}, \nu\right)$ is a graded symplectic manifold $\mathfrak{F}$, equipped with a symplectic form $\omega_{-1}$ of degree -1 and a berezinian volume $\nu$ such that $C(\mathfrak{F})$ is $B V$ algebra.

Let $\Delta_{\nu}$ be the BV-laplacian induced by $\nu$ as

$$
\Delta_{\nu} f=\frac{(-1)^{\operatorname{deg}(f)}}{2} \operatorname{div}_{\nu} X_{f}
$$


for $f \in C(\mathfrak{F})$ and $X_{f}=\{f, \cdot\}$ its hamiltonian vector field. The ring of global functions $C(\mathfrak{F})$ is a (-1)-Poisson algebra and the BV laplacian $\Delta_{\nu}$ is a generator of the odd Poisson bracket satisfying $\Delta_{\nu}^{2}=0$, so that it is a $B V$-algebra. A function $S \in C(\mathfrak{F}), \operatorname{deg} S=0$, solves the Quantum Master Equation (QME) if $\Delta_{\nu} e^{\frac{i}{\hbar} S}=0$. The QME can be equivalently written as

$$
\{S, S\}-2 i \hbar \Delta_{\nu} S=0 .
$$

The equation $\{S, S\}=0$ is called the Classical Master Equation (CME).

This is the geometric setup encoding the BV-quantization [3, 4], as formulated in [32]. For every lagrangian submanifold $\mathcal{L} \subset \mathfrak{F}$ the berezinian $\nu$ restricts to the berezinian $\nu_{\mathcal{L}}$ on $\mathcal{L}$. In 32] it is shown that for each $f \in C(\mathfrak{F})$ such that $\Delta_{\nu} f=0$ and for two cobordant lagrangian submanifolds $\mathcal{L}, \mathcal{L}^{\prime} \subset \mathfrak{F}$ one has

$$
\int_{\mathcal{L}} f=\int_{\mathcal{L}^{\prime}} f \quad ;
$$

while for each $g \in C(\mathfrak{F})$

$$
\int_{\mathcal{L}} \Delta_{\nu} g=0
$$

To briefly illustrate it, let us consider a finite dimensional example. Let $N$ be a finite dimensional smooth compact manifold and consider the cotangent bundle $\mathfrak{F}=T^{*}[-1] N$ equipped with the canonical symplectic form. This corresponds to the canonical odd Poisson bracket $\{$,$\} of degree +1$ (Schoutens bracket on multivectors on $M$ ); and homological charges are degree zero functions $S \in C\left(T^{*}[-1] N\right)$. The choice of a volume form $\lambda$ on $N$ defines a berezinian $\nu_{\lambda}=\lambda \otimes \lambda \in \Gamma\left(\operatorname{Ber}\left(T^{*}[-1] N\right)\right)=\Gamma\left(\operatorname{det}\left(T^{*} N\right) \otimes \operatorname{det}\left(T^{*} N\right)\right)$. For any submanifold $C \subset N, \sqrt{\nu_{\lambda}}:=\left.\lambda\right|_{C}$ defines a berezinian for the lagrangian submanifold $N^{*}[-1] C \subset T^{*}[-1] N$, since $\operatorname{Ber}\left(N^{*}[-1] C\right)=\operatorname{det}\left(N^{*} C\right) \otimes \operatorname{det}\left(T^{*} C\right)=\operatorname{det}\left(\left.T^{*} N\right|_{C}\right)$. The fundamental BV-theorems above are then a reformulation of the Stokes theorem on $N$ (e.g., for a brief review see [29]).

\subsection{The AKSZ construction}

The most interesting examples of $B V$ manifolds come as spaces of fields of Quantum Field Theory. In this case they are infinite dimensional spaces, so that the properties concerning integration must be implemented after renormalization. The AKSZ construction is a method introduced in [1] that gives a solution of the classical master equation starting from very simple geometrical data. We describe it here following [30].

Let us consider the following data: 
i) The source: A graded manifold $\mathcal{N}=T[1] N$, for any smooth oriented manifold $N$ of dimension $n+1$, with $D=d$ being the de Rham differential over $N$ and $\mu$ the canonical Berezinian measure defined by orientation.

ii) The target: A graded symplectic manifold $(\mathcal{M}, \omega)$ with $\operatorname{deg}(\omega)=n$ and an homological vector field $Q$ preserving $\omega$. We require that $Q$ is Hamiltonian, i.e. it exists $\Theta \in C_{n+1}(\mathcal{M})$ (functions of degree $n+1$ ) such that $Q=\{\Theta,-\}$. Therefore $\Theta$ satisfies the following Maurer-Cartan equation

$$
\{\Theta, \Theta\}=0
$$

Remark 2 In the above data, the source can be more general than a graded manifold; what is actually needed is a sheaf of finitely generated Frobenius algebras, see [7] for details and the end of this subsection for the discussion of the AKSZ theory of zero modes.

The space of maps $\mathfrak{F}=\operatorname{Map}(\mathcal{N}, \mathcal{M})$ defines the space of $B V$-fields for the theory (see below), where the BV structure $\left(C(\mathfrak{F}),\{,\}_{\mathfrak{F}}, \Delta\right)$ is given as follows. First, if $\left\{u^{\alpha}, \theta^{\alpha}\right\}$ is a set of coordinates for $T[1] N$, then for any $f \in C(\mathcal{M})$ we have

$$
e v^{*} f=\sum_{I} f_{(I)}(u) \theta^{I}
$$

where $e v: \operatorname{Map}(\mathcal{N}, \mathcal{M}) \times \mathcal{N} \rightarrow \mathcal{M}$ denotes the evaluation map [12], $\theta^{I}=\theta^{\alpha_{1}} \ldots \theta^{\alpha_{k}}$ is a local basis for the fibers of $T[1] \mathcal{N}$. In particular, if $\left\{X^{A}\right\}$ are coordinates for $\mathcal{M}$, a superfield in $\mathfrak{F}$ is the collection $\Phi=\left\{\Phi^{A}\right\}$, where

$$
\Phi^{A}=\Phi_{0}^{A}(u)+\Phi_{\alpha}^{A}(u) \theta^{\alpha}+\Phi_{\alpha_{1} \alpha_{2}}^{A}(u) \theta^{\alpha_{1}} \theta^{\alpha_{2}}+\ldots .
$$

The odd Poisson bracket $\{,\}_{\mathfrak{F}}$ on $\mathfrak{F}$ comes from the symplectic form

$$
\omega_{\mathfrak{F}}=\int_{\mathcal{N}} e v^{*} \omega
$$

and the BV-laplacian is formaly given by $\Delta \simeq \frac{\partial^{2}}{\partial \Phi_{i} \partial \Phi^{i}}$ for Darboux coordinates $\left\{X^{A}=\right.$ $\left.x^{i}, p_{i}\right\}$ on the target $\mathcal{M}$. Notice that the laplacian is in general ill-defined and needs appropriate regularization.

The AKSZ action reads as

$$
S[\Phi]=S_{k i n}[\Phi]+S_{i n t}[\Phi]=\int_{\mathcal{N}} \mu\left(\frac{1}{2} \Phi^{A} \omega_{A B} D \Phi^{B}+(-1)^{n+1} \Phi^{*}(\Theta)\right)
$$

and solves the CME. We refer to this construction as $A K S Z(\mathcal{N},(\mathcal{M}, \omega, \Theta))$. 
If the grading of $\mathcal{M}$ is non negative, then from [30] we have a characterization of the encoded target geometry. For $n=1$ the target manifold is $\mathcal{M}=T^{*}[1] M$, where $M$ is an ordinary smooth manifold and $\Theta=\pi \in C_{2}(\mathcal{M})=\Gamma\left(\Lambda^{2} T M\right)$ is a Poisson structure. If we choose local coordinates $\left\{x^{i}, b_{i}\right\}$ then $\Theta=\frac{1}{2} \pi^{i j} b_{i} b_{j}$. The AKSZ action defines what is known as Poisson Sigma Model (PSM in short). For $n=2$ then the target is $\mathcal{M}(E)$ the graded even symplectic manifold associated to any vector bundle $E$ with nondegenerate symmetric bilinear pairing and $\Theta$ is the hamiltonian associated to a Courant algebroid structure on it. Let us choose coordinates $\left\{x^{i}\right\}$ of degree 0 on $M$ with momenta $\left\{p_{x^{i}}\right\}$ of degree 2 , and a trivialization $\left\{e_{A}\right\}$ of $E$ of orthonormal sections with coordinates $\left\{\lambda^{A}\right\}$ of degree 1. Denote also with $P_{A}^{i}$ and $T_{A B C}$ the coefficients of the anchor and the bracket of the underlying Courant algebroid, respectively. We have that

$$
\Theta=\lambda^{A} P_{A}^{i} p_{x^{i}}-\frac{1}{6} T_{A B C} \lambda^{A} \lambda^{B} \lambda^{C} .
$$

We will refer to the TFT defined by the AKSZ action as the Courant Sigma Model, (CSM in short).

The choice of a Lagrangian $\mathfrak{L} \subset \mathfrak{F}$ inside the space of BV-fields is called gauge fixing. A quantum observable is an $F \in C(\mathfrak{F})$ satisfying $\Delta\left(F e^{i S_{B V} / \hbar}\right)=0$. For such an $F \in C(\mathfrak{F})$, one is interested in computing the integral expression

$$
\langle F\rangle:=\int_{\mathfrak{L}} F e^{\frac{i}{\hbar} S_{B V}},
$$

which is called the (quantum) correlator. As a consequence of the fundamental results of BV quantization, the correlators of quantum observables are independent of the gauge fixing choice.

A finite dimensional analogue of this construction is the $B V$ theory of zero modes. It can be seen as the symplectic reduction of the full theory with respect to the constraint $D \Phi=0$. Indeed, this defines a coisotropic submanifold $\mathfrak{Z}$ of the space of superfields $\mathfrak{F}$ such that the corresponding symplectic reduced space $\mathfrak{F}^{Z}=\mathfrak{F} / / \mathfrak{Z}$ is finite dimensional and inherits all the BV-structure from $\mathfrak{F}$. This reduced space $\mathfrak{F}^{Z}$ is the $B V$-space of zero modes. When $\partial N=\emptyset$, the reduced theory on the space of zero modes can be described again as an AKSZ construction where one takes as source the de-Rham cohomology $H_{d R}(N)$ equipped with zero differential. For further details see [6, 7].

Finally, we recall a useful concept of effective action, see [25] and [28]. Let us consider a $B V$-manifold $\mathcal{F}$ that can be written as a product of $B V$-manifolds $\mathcal{F}_{1} \times \mathcal{F}_{2}$, such that $\Delta=\Delta_{1}+\Delta_{2}$. Let $S \in C(\mathcal{F})$ be a solution of the quantum master equation (QME) $\Delta \exp i S / \hbar=0$. Let $\mathcal{L}_{2}$ be a Lagrangian submanifold of $\mathcal{F}_{2}$ and let us define the effective 
action $S_{\text {eff }} \in C\left(\mathcal{F}_{1}\right)$ as

$$
e^{\frac{i}{\hbar} S_{\text {eff }}}=\int_{\mathcal{L}_{2}} e^{\frac{i}{\hbar} S} .
$$

We clearly have that $S_{\text {eff }}$ solves QME on $\mathcal{F}_{1}$. Indeed we have that

$$
\Delta_{1} e^{\frac{i}{\hbar} S_{\text {eff }}}=\int_{\mathcal{L}_{2}} \Delta_{1} e^{\frac{i}{\hbar} S}=\int_{\mathcal{L}_{2}} \Delta e^{\frac{i}{\hbar} S}=0 .
$$

We call $\mathcal{F}_{1}$ the space of infrared degrees of freedom and $\mathcal{F}_{2}$ the space of ultraviolet ones. For instance if we take $\mathcal{F}$ as the space of superfields of the AKSZ construction and consider the splitting induced by the Hodge decomposition of $N$, where the infrared variables are the coefficient of the cohomology of $N$, we get that $\mathcal{F}_{1}=\mathfrak{F}^{Z}$ the $B V$-space of zero modes. Moreover the reduced BV-action is the lowest order of the expansion of the effective action. When the target $\mathcal{M}$ is a general graded manifold and not just a vector space, then covariance with respect to the change of variables must be taken into account, see [5].

\section{The $B F V_{n}$ manifold}

In this Section we introduce the BFV (Batalin-Fradkin-Vilkovisky) manifold. We give first an axiomatic definition modeled on the usual BFV construction of first class constraints. The main reason for this choice is to emphasize the ingredients that will be used in the AKSZ formulation, rather than all the details of the construction that will be sketched in Section 3.1.

Definition $3 A B F V_{n}$ manifold is a triple $\left(\mathcal{M}, \Omega_{n}, \Theta\right)$, where $\mathcal{M}$ is a $\mathbb{Z}$-graded symplectic manifold with a degree $n$ symplectic form $\Omega_{n}$ and degree $n+1 B F V$-charge $\Theta \in C^{n+1}(\mathcal{M})$ that satisfies $\{\Theta, \Theta\}=0$. Moreover $\mathcal{M}$ is endowed with an extra $\mathbb{Z}$-grading ga called the ghost-antighost degree, such that $g a\left(\Omega_{n}\right)=0$ and $\Theta=\sum_{k \leq 1} \Theta_{k}$, with $g a\left(\Theta_{k}\right)=k$.

In the above definition we implicitly assumed that the $\mathbb{Z}$-degree deg determines the parity $\mathbb{Z}_{2}$-degree of $\mathcal{M}$ seen as a supermanifold. By having an extra $\mathbb{Z}$-grading we mean that there is an atlas of coordinates homogeneous in the ga degree and such that the changes of coordinates also respect this grading. Moreover we assumed the vanishing of the coefficients $\Theta_{r}$ for $g a$ degree $r \geq 2$; all the examples we will consider satisfy this property. 
Example 4 Let $\mathcal{M}$ be a $\mathbb{Z}$-graded manifold with degree 0 symplectic form $\Omega_{0}$ and degree 1 homological hamiltonian $\Theta \in C^{1}(\mathcal{M})$. It is easy to see that $\left(\mathcal{M}, \Omega_{0}, \Theta\right)$ is an example of a $B F V_{0}$ manifold in the above sense if we take the extra grading to be ga=deg. This encompasses the usual versions of the BFV construction (see [23, 31, 34]). Also notice that some of these examples arise when $\mathcal{M}$ is infinite dimensional, like the one obtained by applying the AKSZ construction to the boundary $\partial N$ of the source $N$ as done in [13].

Let us denote with $\mathcal{M}_{0}$ and with $\mathcal{M}_{>}$the submanifolds of $\mathcal{M}$ obtained by putting to zero the coordinates with non zero and negative ga degree, respectively. We have clearly $\mathcal{M}_{0} \subset \mathcal{M}_{>} \subset \mathcal{M}$. The restriction for the $B F V$-charge to have ga-degree less than 2 is motivated by Lemma 8 and by the examples that we will discuss, see in particular Propositions 11 and 21 .

Lemma $5 \mathcal{M}_{0} \subset \mathcal{M}$ is a symplectic submanifold.

Proof. Let $\Pi=\Omega_{n}^{-1}$ be the Poisson tensor. One immediately see that $\left.\Pi\right|_{\mathcal{M}_{0}}$ does not mix coordinates with zero and non zero ga degree, so that $\left.\Omega\right|_{\mathcal{M}_{0}}$ is non degenerate.

Unlike the usual BFV-construction, in general, the ghost-antighost coordinates are not fibre coordinates of some vector bundle over the unconstrained $\mathcal{M}_{0}$, i.e. non linear transformation rules are allowed, as in the next example.

Example 6 The graded manifold description of standard Courant algebroid gives an example of $B F V_{2}$-manifold. Let $\mathcal{M}=T^{*}[2] T[1] M$, with Darboux coordinates $\left(x^{\mu}, b_{\mu}, \psi^{\mu}, p_{\mu}\right)$ with deg $(0,1,1,2)$ and hamiltonian $\Theta=\psi^{\mu} p_{\mu}$. The ga degree is defined as

$$
g a\left(x^{\mu}, b_{\mu}, \psi^{\mu}, p_{\mu}\right)=(0,-1,1,0)
$$

so that $\Theta=\Theta_{1}$ and $\mathcal{M}_{0}=T^{*}[2] M$. Notice that the nonlinear change of coordinates in this case is $p_{\mu} \mapsto p_{\mu}+A_{\mu \lambda}^{\nu}(x) b_{\nu} \psi^{\lambda}$, which indeed respects the extra ga-degree.

We denote with $C^{k}(\mathcal{M})$ the set of functions of $\operatorname{deg}=k$ and with $C_{p}^{k}(\mathcal{M})$ those with $g a=p$ and $\operatorname{deg}=k$. We thus write $Q=\sum_{r \leq 1} Q_{r}$ the hamiltonian vector field, where $Q_{r}=\left\{\Theta_{r},-\right\}: C_{p}^{k}(\mathcal{M}) \rightarrow C_{p+r}^{k+1}(\mathcal{M})$, and with $H_{Q}$ its cohomology.

The local model for $\mathcal{M}$ reads as follows: there are coordinates in which $\Omega_{n}=d x^{\mu} d p_{\mu}+$ $d \xi^{a} d p_{a}$, where $g a\left(x^{\mu}\right)=g a\left(p_{\mu}\right)=0$ and $g a\left(\xi^{a}\right)=-g a\left(p_{a}\right)>0$. We call $\xi^{a}$ the $\left(B F V_{n}\right)$ ghosts and $p_{a}$ the $\left(B F V_{n}\right)$ antighosts. The submanifold $\mathcal{M}_{0} \subset \mathcal{M}$ is locally defined by $p_{a}=\xi^{a}=0$ and $\mathcal{M}_{>}$by $p_{a}=0$.

Let us assume for simplicity that $g a\left(\xi^{a}\right)=-g a\left(p_{a}\right)=1$. The most general case of higher $g a$ degree ghosts and antighosts is related to the reducibility of the constraints of 
the associated reduction data (see below) and these coordinates should be called called ghosts-for-ghosts.

We now analyze the content of the $B F V_{n}$-charge. If we decompose the equation $\{\Theta, \Theta\}=0$ in the ga degree we get for each $r \leq 2$

$$
\sum_{k+\ell=r}\left\{\Theta_{k}, \Theta_{\ell}\right\}=0
$$

If we develop $\Theta_{k}$ on the ghosts/antighosts variables, i.e. $\Theta_{0}=\theta+\theta_{b}^{a} \xi^{b} p_{a}+\ldots, \Theta_{1}=$ $\Theta_{1, a} \xi^{a}+1 / 2 \Theta_{1, a b}^{c} \xi^{a} \xi^{b} p_{c}+\ldots$, we get the following relations on $\mathcal{M}_{0}$

$$
\begin{gathered}
\{\theta, \theta\}+2(-)^{t_{a}} \Theta_{-1}^{a} \Theta_{1, a}=0, \quad\left\{\theta, \Theta_{1, a}\right\}+(-)^{t_{a} t_{b}} \theta_{a}^{b} \Theta_{1, b}=0, \\
\left\{\Theta_{1, a}, \Theta_{1, b}\right\}+\Theta_{1, c} \Theta_{1, a b}^{c}=0,
\end{gathered}
$$

where $t_{a}=\operatorname{deg} \Theta_{1 a}$. If $Q=Q_{1}$, as in the usual $B F V$-construction, then the complex $(C(\mathcal{M}), Q)$ is bigraded by the $g a$-degree.

Definition 7 Degree $n$ reduction data are given by a quadruple $\left(\mathcal{M}_{0}, \omega_{n}, \mathcal{I}, \theta\right)$, where $\mathcal{M}_{0}$ is a graded manifold with symplectic form $\omega_{n}$ of degree $n, \mathcal{I} \subset C\left(\mathcal{M}_{0}\right)$ is a coisotropic ideal, i.e. $\{\mathcal{I}, \mathcal{I}\} \subset \mathcal{I}$, and $\theta \in C^{n+1}\left(\mathcal{M}_{0}\right)$ is a function invariant under the coisotropic distribution and homological on the constraint, i.e. $\{\theta, \mathcal{I}\} \subset \mathcal{I}$ and $\{\theta, \theta\} \in \mathcal{I}$.

The reduced algebra $\left(C^{\infty}\left(\mathcal{M}_{0}\right) / \mathcal{I}\right)^{i n v}$ is a $n$-Poisson algebra; the hamiltonian $\theta$ defines $\theta_{\text {red }} \in\left(C^{\infty}\left(\mathcal{M}_{0}\right) / \mathcal{I}\right)^{i n v}$ and let $Q_{\text {red }}=\left\{\theta_{\text {red }},-\right\}$ satisfying $Q_{\text {red }}^{2}=0$. Let us denote with $H_{Q_{\text {red }}}$ its cohomology. We call $f \in C\left(\mathcal{M}_{0}\right)$ a reducible observable if $\{\theta, f\} \in \mathcal{I}$ and $\{\mathcal{I}, f\} \subset \mathcal{I}$.

We shall say that the reduction data are regular if the ideal $\mathcal{I}$ defines a coisotropic submanifold $\mathcal{C} \subset \mathcal{M}_{0}$ and the quotient $q: \mathcal{C} \rightarrow \mathcal{M}_{0} / / \mathcal{C}$ defines a smooth submersion. In this case, the reduction data induce a degree $n$ symplectic structure $\omega_{\text {red }}$ and an hamiltonian homological vector field $Q_{\text {red }}$ on the reduced graded manifold $\mathcal{M}_{0} / / \mathcal{C}$. In the regular case we denote the reduction data also with $\left(\mathcal{M}_{0}, \omega_{n}, \mathcal{C}, \theta\right)$.

Lemma $8 A B F V_{n}$ manifold $\left(\mathcal{M}, \Omega_{n}, \Theta\right)$ defines the degree $n$ reduction data $\left(\mathcal{M}_{0},\left.\Omega_{n}\right|_{\mathcal{M}_{0}}\right.$, $\left.\mathcal{I}_{\Theta_{1}}, \theta=\left.\Theta\right|_{\mathcal{M}_{0}}\right)$, where $\mathcal{I}_{\Theta_{1}}$ is locally generated by $\Theta_{1, a}$.

Proof. First, notice that $\Theta_{1, a}=\left.\frac{\partial}{\partial \xi^{a}} \Theta_{1}\right|_{\mathcal{M}}$ transforms covariantly under change of coordinates and thus $\mathcal{I}_{\Theta_{1}}$ is globaly well defined. The equations (3.5) imply that $\mathcal{I}_{\Theta_{1}}$ is coisotropic and that $\theta$ is an invariant homological function. 
Given the reduction data $\left(\mathcal{M}_{0}, \omega_{n}, \mathcal{I}, \theta\right)$ and a $\operatorname{BFV}_{n}$ manifold $\left(\mathcal{M}, \Omega_{n}, \Theta\right)$ inducing them, we will say that $\mathcal{M}$ is a $B F V_{n}$-model for the reduction data. $B F V_{n}$-models are in general not unique, see for example [31] for the $n=0$ case.

Let $F=\sum_{r \leq 0} F_{r} \in C(\mathcal{M})$ be concentrated in nonpositive $g a$-degree and $Q$-closed. It is easy to check that $f=\left.F\right|_{\mathcal{M}_{0}}$ is a reducible observable.

Remark 9 In the general case, the correspondence $\left.F \rightarrow F\right|_{\mathcal{M}_{0}}$ does not define a map in cohomology. Let us consider $F+Q(G)$ with $Q(G)_{r}=0$ for $r>0$. Let us denote $G_{0}=g+\ldots, G_{1}=g_{a} \xi^{a}+\ldots, G_{-1}=g^{a} p_{a}+\ldots$ One computes

$$
\left.Q(G)\right|_{\mathcal{M}_{0}}=\{\theta, g\}+(-)^{|g|+\left(\lambda_{a}+1\right)(n+1)} g^{a} \Theta_{1, a}+g_{a} \Theta_{-1}^{a}(-)^{\left.(|g|+1)(n+1)+\lambda_{a} n\right)} \quad .
$$

Even if $\Theta_{-1}^{a}=0$, as it happens in Proposition 21, $g=\left.G\right|_{\mathcal{M}_{0}}$ doesn't automatically define a primitive for $(Q(G))_{\text {red }}$. Indeed, since $Q(G)_{1}=Q_{1} G_{0}+Q_{0} G_{1}+Q_{-1} G_{2}+\ldots=0$, we have in lowest degree in $\xi$

$$
\begin{aligned}
0 & =\left\{\theta, g_{a}\right\}+\left\{\Theta_{1, a}, g\right\}(-)^{\left(n+1+\lambda_{a}\right)(|g|-n)}+\Theta_{1, b} g_{a}^{b}(-)^{\left(1+\lambda_{b}\right)(|g|+1)} \\
& +\theta_{a}^{b} g_{b}(-)^{\lambda_{a} \lambda_{b}+\left(\lambda_{a}+\lambda_{b}\right)(|g|+n+1)}+\Theta_{-1}^{b} g_{b a}(-)^{\left(1+\lambda_{b}\right)(|g|+1)}
\end{aligned}
$$

so that $g$ need not be invariant and does not define a $Q_{\text {red-primitive for }}(Q(G))_{\text {red }}$.

We recall that if $Q=Q_{1}$ then $H_{Q}=\sum_{p, q} H_{Q}^{q, p}$ is bigraded and let us denote $H_{Q}^{g a=0}=$ $\underset{p}{\oplus} H_{Q}^{0, p}$

Proposition 10 If $Q=Q_{1}$, then the map $\left.F \rightarrow F\right|_{\mathcal{M}_{0}}$ for $Q$-closed $F=\sum_{r \leq 0} F_{r}$, concentrated in non positive ga-degree, descends to a map

$$
\Psi: H_{Q}^{g a=0} \rightarrow\left(C\left(\mathcal{M}_{0}\right) / \mathcal{I}\right)^{i n v}
$$

Proof. With the same notation of Remark 9, the result follows from (3.6) observing that $g$ is invariant and so it reduces to a primitive for $(Q(G))_{\text {red }}$.

More generaly, in the above case, one can compute the cohomology $H_{Q}$ using spectral sequence arguments (see [34] for the $n=0$ case).

\subsection{The $B F V_{n}$ construction from reduction data}

We sketch in this subsection the construction of a $B F V_{n}$-model from the regular reduction data $\left(\mathcal{M}_{0}, \omega_{n}, \mathcal{C}, \theta\right)$. The case $n=0$ and $\theta=0$ is standard, see [11] for more details on the generic case (see also [27] for the $n=1$ case). 
In general, this $B F V_{n}$ construction will also depend on non-canonical choices such as a tubular neighbourhood and a connection on the normal bundle to $\mathcal{C}$, as for $n=0$ (see for instance [31]). In this paper, however, we will only consider cases in which $\mathcal{C}$ is given as zero level set of a map $\mu: \mathcal{M}_{0} \rightarrow W$ where $W$ denotes a graded vector space. We shall also make the regularity assumption 11 that $0 \in W$ is a regular value for $\mu$, so that $\left(\mathcal{M}_{0}, \omega_{n}, \mu^{-1}(0), \theta\right)$ define a set of regular reduction data. Our $B F V_{n}$ construction thus depends explicitly on the map $\mu$ chosen.

After choosing a basis in $W$, the components of the moment map $\mu_{a}$ satisfy

$$
\{\theta, \theta\}=\theta^{a} \mu_{a}, \quad\left\{\mu_{a}, \mu_{b}\right\}_{\omega}=\mu_{c} F_{a b}^{c}, \quad\left\{\theta, \mu_{a}\right\}_{\omega}=\theta_{a}^{c} \mu_{c} .
$$

We set the graded manifold $\mathcal{M}=M_{n} \times T^{*}[n] V$ where $V=W^{*}[n+1]$. The Darboux coordinates on $T^{*}[n] V$ are $\left(\xi^{a}, p_{a}\right)$, called ghosts and antighosts respectively, with degrees assignements $\operatorname{deg}\left(\xi^{a}\right)=n+1-\operatorname{deg}\left(\mu_{a}\right)$ and $\operatorname{deg}\left(p_{a}\right)=\operatorname{deg}\left(\mu_{a}\right)-1$ and $g a(\xi)=-g a(p)=1$. The symplectic structure is $\Omega_{n}=\omega_{n} \oplus \varpi_{n}$, where $\varpi_{n}=d \xi^{a} d p_{a}$.

The reduction data fixe the lower terms of the BFV-charge

$$
\begin{gathered}
\Theta_{0}=\theta+\theta_{a}^{c} \xi^{a} p_{c}+O^{2}(p) \\
\Theta_{1}=\mu_{a} \xi^{a}+( \pm) \frac{1}{2} F_{a b}^{c} \xi^{a} \xi^{b} p_{c}+O^{2}(p)
\end{gathered}
$$

so that the equation $\{\Theta, \Theta\}=0$ is satisfied up to second order in the antighosts. The sign in (3.9) can be fixed by comparing it with (3.5).

A full solution for the BFV-charge $\Theta$ can be obtained by using the so called homological perturbation method $([34,[23],[21])$ as follows. Let res be the polynomial degree of the antighosts $p_{a}$ and let $\delta=\mu_{a} \frac{\partial}{\partial p_{a}}$. By degree considerations it is clear that $\delta^{2}=0$; let us denote with $H_{\delta}$ its cohomology. We denote with $F^{(r)}$ the components (of functions and vector fields) with $r e s=r$. Remark that this additional grading is not defined in general for the $B F V_{n}$ space of Definition 3 .

As a consequence of regularity, the homology of $\delta$ is concentrated in degree 0 , i.e. $H_{\delta}^{r}=0$ for $r>0$ (see Theorem 9.1 in [20]).

The proof of the following theorem is standard for the case $\theta=0$ and $n=0$ (see for instance [20]). The general case is similar but observe that terms with negative ga grading appear in $\Theta$ due to the presence of nonvanishing $\theta$.

Proposition 11 i) There exists an homological function $\Theta=\sum_{r \leq 1} \Theta_{r}$ coinciding with (3.9) up to first order in the antighosts.

\footnotetext{
${ }^{1}$ In the non-regular case, one needs to introduce ghosts-for-ghosts of higher $g a$-degree in order to kill unwanted cohomology (see e.g. [34]). The construction goes along similar lines.
} 
ii) For any reducible observable $f \in C\left(\mathcal{M}_{0}\right)$ there exists $Q$-closed $F^{\infty}=\sum_{r} F^{(r)} \in$ $C(\mathcal{M})$, where $F^{(0)}=f$, res $F^{(r)}=r$ and $F^{(r)}=\sum_{l \leq 0} F_{l}^{(r)}$, where $g a\left(F_{l}^{(r)}\right)=l$. This correspondence defines a map

$$
\lambda: H_{Q_{r e d}} \rightarrow H_{Q}
$$

iii) If $\theta=0$ then $Q=Q_{1}$ and $\lambda$ inverts the map $\Psi$ defined in Proposition 10 .

Proof.

i) Let $\Theta^{(0)}=\theta+\mu_{a} \xi^{a}=\Theta_{0}^{(0)}+\Theta_{1}^{(0)}$ and using (3.77) let us compute

$$
\left\{\Theta^{(0)}, \Theta^{(0)}\right\}=-2 \delta \Theta^{(1)}
$$

where $\Theta^{(1)}=\sum_{r=-1}^{1} \Theta_{r}^{(1)}$. Define $\Theta^{\leq 1}=\Theta^{(0)}+\Theta^{(1)}$; by direct computation we get $\operatorname{res}\left(\left\{\Theta^{\leq 1}, \Theta^{\leq 1}\right\}\right) \geq$ 1. This procedure can be iterated. Let $\Theta^{\leq k}=\sum_{l=0}^{k} \Theta^{(k)}$, with $\Theta^{(l)}=\sum_{r \leq 1} \Theta_{r}^{(l)}$, satisfy $\operatorname{res}\left(\left\{\Theta^{\leq k}, \Theta^{\leq k}\right\}\right) \geq k$ and we can thus write $\left\{\Theta^{\leq k}, \Theta^{\leq k}\right\}=r^{(k)}+($ res $\geq k+1)$. Since by Jacobi identity

$$
0=\left\{\Theta^{\leq k},\left\{\Theta^{\leq k}, \Theta^{\leq k}\right\}\right\}=\delta r^{(k)}+(\text { res } \geq k)
$$

we get that $\delta r^{(k)}=0$, so that by regularity assumption $r^{(k)}=-2 \delta \Theta^{(k+1)}$. By taking into account that $g a(\delta)=1$ we also easily see that we can choose $\Theta^{(k+1)}$ such that $\Theta^{(k+1)}=$ $\sum_{r \leq 1} \Theta_{r}^{(k+1)}$. Then $\Theta^{\leq k+1}=\sum_{l=0}^{k+1} \Theta^{(l)}$ satisfies $\operatorname{res}\left(\left\{\Theta^{\leq k+1}, \Theta^{\leq k+1}\right\}\right) \geq k+1$. We can then get $\Theta=\sum_{k \geq 0} \Theta^{(k)}$ by induction.

ii) The BFV charge (cf. eqs. (3.9/3.8)) is decomposed in the res degree as

$$
Q=\delta+\sum_{l \geq 0} Q^{(l)}
$$

Since $f$ is reducible, we have $\{\theta, f\}=f^{a} \mu_{a}$ and $\left\{\mu_{a}, f\right\}=f_{a}^{b} \mu_{b}$, so that one computes

$$
Q f=\{\Theta, f\}=-\delta F^{(1)}+(\text { res } \geq 1),
$$

where $F^{(1)}=F_{0}^{(1)}+F_{-1}^{(1)}$. Define $F^{(\leq 1)}=f+F^{(1)}$ and compute $Q F^{(\leq 1)}=($ res $\geq 1)=$ $r^{(1)}+($ res $\geq 2)$ where $r^{(1)}$ denotes the res $=1$ part of $Q F^{(\leq 1)}$. Thus, from $Q^{2}=0$ we get $0=Q^{2} F^{(\leq 1)}=\delta\left(r^{(1)}\right)+($ res $\geq 1)$ so that $\delta\left(r^{(1)}\right)=0$. By the regularity assumption, there must exist $F^{(2)}$ such that $\delta F^{(2)}=-r^{(1)}$ and res $F^{(2)}=2$. Moreover, since the non vanishing terms of $r^{(1)}$ appear with $g a \leq 1$, then $F^{(2)}$ can be chosen with nonvanishing 
ga-components only for $g a \leq 0$. The procedure can be iterated and adding corrections analogously, we get the representative $F^{\infty}(f)=\sum_{i} F^{(i)}$.

Let now $f_{\text {red }}$ be $Q_{\text {red }}$ exact, i.e. let $f=\{\theta, g\}+\mu_{a} g^{a}$ and $\left\{\mu_{a}, g\right\}=g_{a}^{b} \mu_{b}$. We want to find $G$ such that $F^{\infty}(f)=Q(G)$. The components in res degree must satisfy for each $k \geq 0$

$$
\delta G^{(k)}=F^{(k-1)}-\sum_{r=0}^{k-1} Q^{(k-1-r)}\left(G^{(r)}\right) .
$$

One can check that with $G^{(0)}=g$ and $G^{(1)}=p_{a}\left(g^{a} \pm g_{b}^{a} \xi^{b}\right)$ it is satisfied with $k=0,1$ (the sign can be easily fixed, but it is irrelevant here). We proceed by induction and let us assume that it exists $G^{(l)}, l \leq k$, satisfying (3.11) for $k \geq 1$. In order to show that it is true for $k+1$, by the regularity of the constraint it is enough to show that

$$
\delta\left(F^{(k)}-\sum_{r=0}^{k} Q^{(k-r)}\left(G^{(r)}\right)\right)=0,
$$

which is done by a straightforward computation. Also, the ambiguity involved in choosing $\delta$ potentials translates into $Q$ exact terms, so that we get in cohomology the map (3.10) we wanted.

iii) If $\theta=0$ then it is clear from $i$ ) that $\Theta^{(k)}=\Theta_{1}^{(k)}$ and from $\left.i i\right)$ that $g a\left(F^{\infty}(f)\right)=0$. Moreover, $\Psi \circ \lambda=\mathrm{id}$ is obvious, and $\lambda \circ \Psi=\mathrm{id}$ can be proven similarly.

Remark 12 If $\Theta=\Theta_{1}$, as in the usual BFV construction, then the BFV cohomology is graded by ga-degree and the image of $\Psi^{-1}$ described in Proposition 11 is $H_{Q}^{g a=0}$. As a corollary we get that, in this case, $H_{Q}^{g a=0}=C\left(\mathcal{M}_{0} / / \mathcal{C}\right)$ as graded Poisson algebras.

Remark 13 There is another useful object related to BFV cohomology for ga $\geq 0$, namely its vertical complex (cf [21]). It is given by $\mathcal{V}:=\left(C\left(\left.\mathcal{M}_{>}\right|_{\mathcal{C}}\right), d_{v}\right)$ where $d_{v} \circ r=r \circ Q$, being $r: C(\mathcal{M}) \rightarrow C\left(\left.\mathcal{M}_{>}\right|_{C_{n}}\right)$ the restriction to the submanifold $\left.\mathcal{M}_{>}\right|_{C_{n}} \subset \mathcal{M}$ defined by setting all negative ga coordinates to zero and by restriction to the coisotropic $\mathcal{C} \subset \mathcal{M}_{0}$ for the ga degree zero coordinates. One can use arguments similar to the above proof to show ([11]) that the map $r$ induces an isomorphism from the BFV cohomology of $(C \cdot(\mathcal{M}), Q)$ to the cohomology of $\mathcal{V}$. There is also a map $i: C(\mathcal{C}) \rightarrow \mathcal{V}$ given by the inclusion of the ga degree zero part and one has that the map (3.10) factors as $\lambda=r^{-1} \circ i \circ q^{*}$. It is, in general, the map induced by $i \circ q^{*}$ in cohomology the one that might be non injective.

\section{The AKSZ-BFV system}

We consider here a field theoretical realization of the homological reduction described in the previous sections. We will introduce first spaces that are at the same time $B V$ and 
$B F V_{-1}$. Indeed the case $n=-1$ is special in the $B F V$ construction, since the $B F V_{-1}$ space is a symplectic manifold of degree -1 endowed with a charge of degree zero. The homological condition can be seen as the CME and it is natural to require the existence of a berezinian such that the charge solves the QME too. This is what we will call a $B V$-BFV manifold. We show that it must be seen as an homological model for the reduction of $B V$-manifolds. In subsection 4.1 we discuss the reduction of the most basic example of BV-manifold, $T^{*}[-1] N$, with respect to a group action. In this example we understand concretely how the structure of BV-BFV manifold encodes the reduction of the BV structure. In subsection 4.2 we discuss the case we are most interested in, i.e. the AKSZ construction in dimension $d+1$ having a $B F V_{d}$ manifold as target.

\subsection{BV-BFV manifolds}

Definition $14 A B V-B F V$ manifold is a $B F V_{-1}$ manifold $\left(\mathfrak{F}, \omega_{-1}, \Theta\right)$ endowed with a berezinian $\nu$ (so that it becomes a $B V$-manifold) such that $\Theta$ solves the $Q M E$ and $g a\left(\Delta_{\nu}\right)=$ 0.

This definition implies that on the $B F V$ charge we impose $\Delta_{\nu} \Theta=0$; as a consequence, if $Q=\{\Theta,-\}$, then $\left[Q, \Delta_{\nu}\right]=Q \Delta_{\nu}+\Delta_{\nu} Q=0$ and the cohomology $H_{Q}$ of the $B F V$ differential inherits a generator for its odd Poisson bracket, i.e. it is a $B V$-algebra. Since $g a(\Delta)=0$ then $\mathfrak{F}_{0} \subset \mathfrak{F}$, obtained by putting all ghosts and antighosts to zero, is a $B V$ manifold (or better $C\left(\mathfrak{F}_{0}\right)$ is a $B V$-algebra).

This definition gives an insight into the reduction of $B V$-manifolds. Let $\Theta=\Theta_{1}$ be concentrated in ga degree 1 so that the restriction $\left.F \rightarrow F\right|_{\mathfrak{F}_{0}}$ descends to a map $\Psi: H_{Q}^{g a=0} \rightarrow C\left(\mathfrak{F}_{0} / / C_{\Theta_{1}}\right)$, as in Proposition 10, If the constraints defined by $\Theta$ are regular then $\Psi$ is an isomorphism and $\Delta_{\nu}$ induces a generator of the Poisson bracket on $C\left(\mathfrak{F}_{0} / / C_{\Theta_{1}}\right)$. We want to stress here the role of the regularity condition on the constraints. Indeed the same regularity condition that allows the BFV construction out of reduction data allows the reduction of the BV laplacian. Let us spell out the conditions given by Definition 14, By using the same notations as in Section 3.1, we see that the QME equation for $\Theta$ implies $\Delta_{\nu} \mu_{a}+F_{a b}^{b}=0$. Let $f \in C\left(\mathfrak{F}_{0}\right)$ be reducible, i.e. $\left\{\mu_{a}, f\right\}=f_{a}^{b} \mu_{b}$; if $f$ is in the image of $\Psi$ then the induced $B V$-generator is

$$
\Delta_{\nu}^{\prime} f=\Delta_{\nu} f+f_{a}^{a}
$$

For a generic reducible $f$ this definition depends on the choice of $f_{a}^{a}$. Let $g_{a}^{b}$ satisfying $g_{a}^{b} \mu_{b}=f_{a}^{b} \mu_{b}$, then $\delta\left[\left(g_{a}^{b}-f_{a}^{b}\right) p_{b} \epsilon_{a b}\right]=0$, for $\epsilon_{a b}= \pm 1$, and by the regularity condition we get that $f_{a}^{b}-g_{a}^{b}=c_{a}^{b c} \mu_{c}$. Moreover, one computes that

$$
\left\{\mu_{a}, \Delta_{\nu}^{\prime} f\right\}=(-)^{1+t_{a}}\left(\Delta_{\nu} f_{a}^{b}\right) \mu_{b}+j_{a}(f)
$$


for some $j_{a}(f)$ to be computed explicitly. One checks that the regularity assumption implies that $j_{a}(f)=j_{a}^{b} \mu_{b}$ so that $\Delta_{\nu}^{\prime}$ defines a generator for the Poisson bracket of $C\left(\mathfrak{F}_{0} / / C_{\Theta_{1}}\right)$.

Let us now discuss how to get a $B V-B F V$ manifold starting from the finite dimensional model $\mathfrak{F}_{0}:=T^{*}[-1] N$ for the space of BV-fields considered in Section 2.1 when $N$ is acted by a Lie group $G$. Let $v_{a} \in \mathcal{X}(N)$ be the vector fields generating the $G$ action on $N$. This action can be lifted to $T^{*}[-1] N$ where it becomes hamiltonian with moment map components given by the degree -1 functions $C\left(T^{*}[-1] N\right) \ni \mu_{a} \cong V_{a}$. Then, $\mathfrak{C}_{\mu}:=\left\{\mu_{a}=0\right\} \subset T^{*}[-1] N$ defines a coisotropic submanifold. If the quotient map $q: N \rightarrow N / G$ is a smooth submersion, the coisotropic reduction yields

$$
\mathfrak{F}_{\text {red }}:=T^{*}[-1] N / / \mathfrak{C}_{\mu} \cong T^{*}[-1](N / G) .
$$

Now, let $S_{0} \in C\left(T^{*}[-1] N\right)$ be a degree zero, $G$-invariant $\left(\left\{\mu_{a}, S_{0}\right\}=0\right)$ homological charge, so that $S_{0}$ reduces to $S_{\text {red }} \in C\left(\mathfrak{F}_{\text {red }}\right)=C\left(T^{*}[-1](N / G)\right)$. In this case, the $B F V_{-1}$ construction applied to the regular reduction data $\left(\mathfrak{F}_{0}=T^{*}[-1] N, \omega_{0}, \mathfrak{C}_{\mu}, S_{0}\right)$ yields

$$
\begin{aligned}
(\mathfrak{F} & \left.:=T^{*}[-1](N \times \mathfrak{g}[1]), \omega_{c a n}, S\right) \\
S & =S_{0}+\Theta_{1} \\
\Theta_{1} & =\mu_{a} \xi^{a}-\frac{1}{2} f_{a b}^{c} p_{c} \xi^{a} \xi^{b}
\end{aligned}
$$

with $\xi^{a}$ being coordinates on $\mathfrak{g}[1]$ and $p_{a}$ their $d e g=-2$ conjugates.

The key point is that this $B F V_{-1}$ space can be also endowed with a BV structure as follows. Let us choose a volume form $\lambda$ on $N$ and denote with $\nu_{\lambda}$ the corresponding berezinian; let $\nu_{g h}=c d \xi^{1} \ldots d p_{1} \ldots$ be a berezinian for the ghost part $(c \in \mathbb{R})$ with BV-laplacian $\Delta_{g h}=\frac{\partial^{2}}{\partial p_{a} \partial \xi^{a}}$. The total $B V$-laplacian is $\Delta=\Delta_{\lambda}+\Delta_{g h}$. The QME for $S$ implies $\Delta(S)=0$, i.e. for each $a=1 \ldots \operatorname{dim} \mathfrak{g}$

$$
\Delta_{\lambda}\left(\mu_{a}\right)+f_{a b}^{b}=0 \quad .
$$

Let us introduce the fermionic delta function supported on $\mu^{-1}(0) \subset T^{*}[-1] N$ as

$$
\delta(\mu)=\int_{\mathfrak{S}_{g h}} e^{\frac{i}{\hbar} \Theta_{1}} \sqrt{\nu_{g h}}=c \mu_{1} \ldots \mu_{k}
$$

where $\mathfrak{L}_{g h}=\left\{p_{a}=0\right\} \subset T^{*}[-1] \mathfrak{g}[1]$. Equation (4.12) is equivalent to the existence of a volume form $\lambda_{\text {red }}$ on the quotient $N / G$ satisfying

$$
i_{\delta(\mu)} \lambda=q^{*} \lambda_{\text {red }} .
$$


The above equation defines $\lambda_{\text {red }}$ : remark that it contains the dependence on the choice of the ghost berezinian $\nu_{g h}$ and on $\hbar$. We conclude that, in this case, $\left(T^{*}[-1](N \times\right.$ $\left.g[1]), \omega_{c a n}, \nu_{\lambda} \times \nu_{g h}, S\right)$ is a BV-BFV manifold and the reduced $B V$ manifold is $\left(T^{*}[-1](N / G)\right.$, $\left.\omega_{\text {can }}, \nu_{\lambda_{\text {red }}}\right)$.

Let $F=\sum_{r \leq 0} F_{r} \in C(\mathfrak{F})$ be concentrated in non positive $g a$ degree and be a quantum observable, i.e. $\Delta\left(F e^{i S / \hbar}\right)=0$. This in particular implies that $f$ reduces to $f_{\text {red }}$ and $\Delta_{\lambda} f+f_{a}^{a}=0$, where $F_{0}=f+f_{a}^{a} \xi^{a} p_{a}+\ldots$ The following gives a finite dimensional version of the computation of correlators for the $B V-B F V$ system.

Proposition 15 Let $\tilde{\Gamma} \subset N / G$ be a submanifold such that there exists a $\Gamma \subset N$ with $\left.q\right|_{\Gamma}: \Gamma \rightarrow \tilde{\Gamma}$ a local diffeomorphism. Then,

$$
\langle F\rangle_{\lambda}=\int_{N^{*}[-1] \Gamma \times \mathfrak{L}_{g h}} F e^{\frac{i}{\hbar} S} \sqrt{\nu_{\lambda} \times \nu_{g h}}=\int_{N^{*}[-1] \tilde{\Gamma}} f_{\text {red }} e^{\frac{i}{\hbar} S_{r e d}} \sqrt{\nu_{\lambda_{\text {red }}}}=\left\langle f_{\text {red }}\right\rangle_{\lambda_{\text {red }}}
$$

yields the corresponding integral on $\mathfrak{F}_{\text {red. }}$. Moreover, this integral only depends on $f_{\text {red }}$ and the homology class of the gauge fixing $\tilde{\Gamma}$.

Proof: The following identity yields

$$
\langle F\rangle=\int_{N^{*}[-1] \Gamma \times \mathfrak{L}_{g h}}\left(F e^{\frac{i}{\hbar} S}\right) \sqrt{\nu_{\lambda} \times \nu_{g h}}=\int_{N^{*}[-1] \Gamma}\left(f e^{\frac{i}{\hbar} S_{0}} \delta(\mu)\right) \sqrt{\nu_{\lambda}} .
$$

Recall that [32] the BV laplacian $\Delta_{\lambda}$ on $T^{*}[-1] N$ can be obtained from de Rham differential $d$ on $T[1] N$ via a Fourier-like transform $\mathcal{F}_{\lambda}: C(T[1] N) \rightarrow C\left(T^{*}[-1] N\right)$ for which

$$
\int_{N^{*}[-1] \Gamma} \mathcal{F}_{\lambda}(\alpha) \sqrt{\nu_{\lambda}}=\int_{\Gamma} \alpha
$$

where $\alpha$ is a differential form. Moreover, $\mathcal{F}_{\lambda}^{-1}(g)=i_{g} \lambda$ so that if $g$ is reducible, by using definition (4.13) we get $\mathcal{F}_{\lambda}^{-1}(g \delta(\mu))=q^{*} \mathcal{F}_{\lambda_{\text {red }}}^{-1}\left(g_{\text {red }}\right)$. The result then follows by applying it to $g=f \exp \left(i S_{0} / \hbar\right) \delta(\mu)$ in (4.14).

Remark 16 Notice that the 'gauge fixing' transversality condition on $\Gamma$ with respect to the quotient $q: N \rightarrow N / G$ is necessary for $\langle F\rangle_{\lambda}$ to be different from zero due to the presence of $\delta(\mu)$.

Remark 17 Recall the Weil model for equivariant cohomology $\left(T[1](N \times \mathfrak{g}[1]), d_{W}\right)$ seen as a $Q$-manifold. Using a volume form $\lambda$ on $N$ satisfying (4.13) and an invariant inner product on $\mathfrak{g}$, one can define a formal Fourier-like transform to the BFV space $\mathcal{F}$ : 
$C(T[1](N \times \mathfrak{g}[1])) \rightarrow C\left(T^{*}[-1](N \times \mathfrak{g}[1])\right)$. It sends the Weil differential $d_{W}$ to a laplacian $\Delta^{B F V}$ given by $\Delta^{B F V}=\Delta_{\lambda}+\frac{\partial^{2}}{\partial p_{a} \partial \xi^{a}}+\left\{\Theta_{1}, \cdot\right\}$ where $\Theta_{1}$ is the pure $B F V$ charge given above. This establishes a clear relation between the Weil model for equivariant de Rham cohomology together with integration of basic forms on the one hand and the $B F V_{-1}$ model as a BV-manifold together with integration of the observables given in the above Lemma, on the other.

Remark 18 Similar constructions to the BV-structure on $\mathfrak{F}$ presented in this section, were considered in [37 for the cases of hamiltonian group actions on BV-algebras. On the other hand, in that reference the focus is on the associated $\mathfrak{g}$-invariant cohomology rather than on the BFV-cohomology which is the one that leads to functions on the reduced space $\mathfrak{F}_{\text {red }}$ as studied here.

\subsection{General features}

A $B F V_{n}$ space can be used as a target for the AKSZ construction. In fact a $B F V_{n}$ space is, in particular, a degree $n$-symplectic manifold endowed with an homological hamiltonian of degree $n+1$, which is the piece of data needed for the target space of the AKSZ construction of an $n$-dimensional topological field theory. We call it the $B F V-A K S Z$ model. By construction, the corresponding space of fields $\mathfrak{F}$ will inherit both BFV and BV structures and becomes a $B V-B F V$ manifold as described in Section 4.1.

In this section we discuss some general features of the BFV-AKSZ model; the discussion will be rather formal at the field space level and some of the statements will become precise once that we discuss finite dimensional examples. The main point is to sketch how this BFV-AKSZ system can be used as a model for the AKSZ system defined by the underlying reduced target geometry $\mathcal{M}_{0} / / C_{\Theta_{1}}$. Namely, reduction-data-compatible computations using the BFV-AKSZ system can be used to obtain the corresponding computations in the reduced theory.

Let $\left(\mathcal{M}, \Omega_{n}, \Theta\right)$ be the $B F V_{n}$ space with $\Theta=\sum_{r \leq 1} \Theta_{r}$ defining the regular degree $n$ reduction data $\left(\mathcal{M}_{0},\left.\Omega_{n}\right|_{\mathcal{M}_{0}}, C_{\Theta_{1}}, \theta=\left.\Theta\right|_{\mathcal{M}_{0}}\right)$ be as in Lemma 8 . We shall keep the notation involving the reduction data from Section 4 . Consider the BV field spaces defined by

$$
\begin{gathered}
\mathfrak{F}_{0}=\operatorname{Map}\left(T[1] \Sigma_{n+1}, \mathcal{M}_{0}\right), \quad \mathfrak{F}_{\text {red }}=\operatorname{Map}\left(T[1] \Sigma_{n+1}, \mathcal{M}_{0} / / C_{\Theta_{1}}\right) \\
\mathfrak{F}=\operatorname{Map}\left(T[1] \Sigma_{n+1}, \mathcal{M}\right) .
\end{gathered}
$$


The field spaces above are endowed with anti-brackets of degree +1 and with homological charges given by the AKSZ actions $S_{\theta}, S_{\theta_{r e d}}$ and $S_{\Theta}$, respectively, as described in section 2.2. We shall proceed formally by treating them as finite dimensional manifolds (this will indeed be the case for zero modes AKSZ theories [7] discussed in Subsection 5.3 and Appendix E).

The choice of local Darboux coordinates $\left(x^{\mu}, p_{\mu}, \xi^{a}, p_{a}\right)$ on $\mathcal{M}$, as described in Section 4 , defines the superfields $\left(\boldsymbol{x}^{\mu}, \boldsymbol{p}_{\mu}, \boldsymbol{\xi}^{a}, \boldsymbol{p}_{a}\right)$. In particular we call the components of $\boldsymbol{\xi}$ and $\boldsymbol{p}$ the (BFV) ghosts and antighosts. The coisotropic $C_{\Theta_{1}} \subset \mathcal{M}_{0}$ gives rise to what can be called a first class constraint AKSZ system on $\mathfrak{F}_{0}$, by considering the coisotropic submanifold $\mathfrak{C}_{\Theta_{1}}=\operatorname{Map}\left(T[1] \Sigma_{n+1}, C_{\Theta_{1}}\right) \subset \mathfrak{F}_{0}$. It can be defined as the vanishing of all the components $\Theta_{1, a I}$ of

$$
\Theta_{1, a}(\boldsymbol{x}, \boldsymbol{p})=e v^{*} \Theta_{1, a}=\sum_{I} \Theta_{1, a I} \theta^{I}
$$

expanded as in eq. (2.1). Moreover, if the symplectic potential 1 -form $\vartheta_{\mathcal{M}_{0}}$ on $\mathcal{M}_{0}$ is also reducible by $C_{\Theta_{1}}$ one can say that $A K S Z$ commutes with reduction in the sense that

$$
\mathfrak{F}_{0} / / \mathfrak{C}_{\Theta_{1}}=\mathfrak{F}_{\text {red }}
$$

together with their QP-structure. In particular, $\left.S_{\theta}\right|_{\mathfrak{C}}=\tilde{q}^{*} S_{\theta_{\text {red }}}$ where $\tilde{q}: \mathfrak{C}_{\Theta_{1}} \rightarrow \mathfrak{F}_{0} / / \mathfrak{C}_{\Theta_{1}}$ denotes the quotient map.

Moreover, one could formally repeat the $B F V_{-1}$ construction of section 4.1 to the reduction data $\left(\mathfrak{F}_{0}, \mathfrak{C}_{\Theta_{1}}, \boldsymbol{\omega}_{-1}, S_{\theta}\right)$ at the field space level and show that AKSZ commutes with $B F V$. By this we mean that the construction in which one first builds the $B F V_{n}$ for the target and then applies the AKSZ construction, namely $\mathfrak{F}$, is a $B F V_{-1}$ model for the above reduction data on $\mathfrak{F}_{0}$. This statement can be made precise in the finite dimensional setting of zero modes AKSZ theories, see Appendix E for details.

Let us now analyze the BV structure of the system defined by $S_{\Theta}=\sum_{r \leq 1} S_{\Theta_{r}}$.

In the case $\mathfrak{F}=\mathfrak{F}_{0} \times \mathfrak{F}_{\text {ghosts }}$, for example, when $\mathcal{M}$ comes from the BFV construction of sect. 3.1, let us consider a BV laplacian $\Delta$ on $C(\mathfrak{F})$ given by

$$
\Delta=\Delta_{0}+\Delta_{g h}
$$

where $\Delta_{0}$ is a BV laplacian on $\mathfrak{F}_{0}$ and $\Delta_{g h} \simeq \frac{\partial^{2}}{\partial p_{a} \partial \xi^{a}}$ is a laplacian acting on the ghost variables. For general $\mathfrak{F}$, we require that $g a(\Delta)=0$ as in the above case. We know that $S_{\Theta} \in C(\mathfrak{F})$ satisfies the CME by construction and we shall further assume that the QME holds so that $\Delta\left(S_{\Theta}\right)=0$. Notice that this last equation can be expanded in $g a$-degrees in which each term has to be zero. In the toy model of section 4.1, we saw that the $g a=1$ term implied the reducibility of the volume form from $\mathfrak{F}$ to $\mathfrak{F}_{\text {red }}$. 
Let us now consider a gauge fixing $\mathfrak{L} \subset \operatorname{Map}\left(T[1] \Sigma_{n+1}, \mathcal{M}_{>}\right)$, i.e. in the ghost sector we put to zero the antighosts $\boldsymbol{p}_{a}=0$. With this choice $\left.S_{\Theta}\right|_{\mathfrak{L}}=S_{\theta}-\int_{T[1] \Sigma} \boldsymbol{\xi}^{a} \Theta_{1, a}(\boldsymbol{x}, \boldsymbol{p})$, so that the ghosts $\boldsymbol{\xi}$ appear as Lagrange multipliers for the constraints $\Theta_{1, a I}$ on $\mathfrak{F}_{0}$. Notice that when $\mathfrak{F}=\mathfrak{F}_{0} \times \mathfrak{F}_{\text {ghosts }}$, the ghosts and antighosts can be interpreted as ultraviolet degrees of freedom in the spirit of the effective action described in Section 2.2. The UV gauge fixing $\mathfrak{L}_{\text {ghost }}=\left\{\boldsymbol{p}_{a}=0\right\}$ thus yields as the effective action

$$
e^{\frac{i}{\hbar} S_{e f f}}=\int_{\mathfrak{L}_{\text {ghost }}} e^{\frac{i}{\hbar} S_{\Theta}}=e^{\frac{i}{\hbar} S_{\theta}} \delta\left(\Theta_{1, a}(\boldsymbol{x}, \boldsymbol{p})\right),
$$

i.e. the AKSZ action restricted to the constraint $\mathfrak{C}_{\Theta_{1}}$.

Remark 19 Recall that for a coisotropic ideal $I \subset C\left(\mathfrak{F}_{0}\right)$, the Poisson reduction is defined as $C\left(\mathfrak{F}_{\text {red }}\right):=N(I) / I$ where $N(I)=\left\{f \in C\left(\mathfrak{F}_{0}\right):\{I, f\} \subset I\right\}$ are the reducible functions. Then, for $f \in N(I)$, one formaly has that $f \delta\left(\Theta_{1, a}(\boldsymbol{x}, \boldsymbol{p})\right) \equiv f_{\text {red }}$ since multiplying by the delta function is equivalent to perform the quotient by I. In particular, for the effective action above we have $S_{\text {eff }} \equiv S_{\theta_{\text {red }}}$.

Let $F=\sum_{r \leq 0} F_{r} \in C(\mathcal{M})$ be a classical observable concentrated in nonpositive $g a$ degree, for instance one in the image of the map (3.10). We recall that $f=\left.F\right|_{\mathcal{M}_{0}}$ is a reducible observable that reduces to $f_{\text {red }} \in C\left(\mathcal{M}_{0} / / C_{\Theta_{1}}\right)$. Denoting $O_{F}=F\left(\boldsymbol{x}^{\mu}, \boldsymbol{p}_{\mu}, \boldsymbol{\xi}^{a}, \boldsymbol{p}_{a}\right)$; we then have that

$$
\left.O_{F}\right|_{\mathfrak{F} \times \mathfrak{L}_{\text {ghost }}}=O_{f}=f(\boldsymbol{x}, \boldsymbol{p}) \in C(\mathfrak{F}) .
$$

Assuming that $\Delta O_{F}=0$, let us compute the expectation values on the extended BV system,

$$
\begin{aligned}
\left\langle O_{F}\right\rangle_{\mathcal{L}} & =\int_{\mathfrak{L}} O_{F} e^{\frac{i}{\hbar} S_{\Theta}} \\
& =\int_{\mathfrak{L}_{0} \times \mathfrak{L}_{\text {ghost }}} O_{f} e^{\frac{i}{\hbar} S_{\theta}-i \int_{T[1] \Sigma} \boldsymbol{\xi}^{a} \Theta_{1, a}(\boldsymbol{x}, \boldsymbol{p})} \\
& =\int_{\mathfrak{L}_{0}} \delta\left(\Theta_{1, a}(\boldsymbol{x}, \boldsymbol{p})\right) O_{f} e^{\frac{i}{\hbar} S_{\theta}} \\
& \left.=: O_{f_{\text {red }}}\right\rangle_{\text {red }}
\end{aligned}
$$

where $\mathfrak{L}_{0} \subset \mathfrak{F}_{0}$ is an IR gauge fixing.

In the toy model of Section 4.1, we showed that the last line above is indeed an equality. In this general infinite-dimensional setting, the last line above must be seen as a definition 
of the expectation value for the theory with reduced target $\left(M_{n} / / C_{n}, \omega_{M_{n} / / C_{n}}, \theta_{M_{n} / / C_{n}}\right)$. Also notice that the above correlator actually depends on the class of $F$ in $H_{Q}$ which, for $\Theta_{0} \neq 0$, can correspond to different classes in $H_{Q_{\text {red }}}$ since the map $\lambda\left(f_{\text {red }}\right)=F^{\infty}(f)$ of Proposition 11 can be non injective (see the discussion in Remark 9).

From the finite dimensional computation in section 4.1, we also notice that the freedom of independently choosing the infrared and ultraviolet gauge fixing is apparent. Indeed the lagrangian $\mathfrak{L}_{0}$ must be transversal to the gauge transformations. This is a point that will be analyzed further in the example of the following Section for the PSM.

Remark 20 The case $n=0$ corresponding to the usual BFV $V_{0}$ construction is discussed in Appendix A. It is shown how, with a slight modification in order to encode non-topological dynamics, the corresponding BFV-AKSZ system yields a BV-quantization procedure of hamiltonian systems with symmetry (see also [18]).

\section{$5 \quad$ Reduction by group actions for $n=1$}

\section{1 $B F V_{1}$ for Poisson actions}

We discuss in this section examples of the $B F V_{1}$ construction outlined in the previous sections for the case of a group action. Nonnegatively graded symplectic manifolds of degree 1 encode Poisson geometry, so that the geometric background of this Section is the reduction of Poisson structures by group action.2. Relevant facts of Poisson Lie geometry are summarized in Appendix B. We will consider first the reduction by the Poisson action of a Poisson Lie group, which is encoded by a degree 1 momentum map. As a final example, we will consider also degree 0 momentum map in the case of an invariant Poisson structure.

Let $\left(M, \pi_{M}\right)$ be a Poisson manifold and let $\left(G, \pi_{G}\right)$ be a Poisson Lie group acting on it with a Poisson action (see Appendix $\mathrm{B}$ for the basic notions on Poisson reduction). Analogous to section 4.1, choosing a basis $\left\{T_{a}\right\}$ of $\mathfrak{g}$ and graded coordinates $\left(x^{i}, b_{i}\right)$ on $T^{*}[1] M$, the fundamental vector fields $v_{a}=v_{a}^{i} \partial_{i}$ define the (degree 1 ) equivariant moment $\operatorname{map} \mu_{G}^{(1)}: T^{*}[1] M \rightarrow \mathfrak{g}^{*}[1]$ for the lifted $G$-action on $T^{*}[1] M$, whose components are

$$
\left(\mu_{G}^{(1)}\right)_{a}=v_{a}^{i} b_{i} \in C^{1}\left(T^{*}[1] M\right) .
$$

Since the action is Poisson, then $\pi_{M} \in C^{2}\left(T^{*}[1] M\right)$ is not invariant but satisfies

$$
\left\{\left(\mu_{G}^{(1)}\right)_{a}, \pi_{M}\right\}=\frac{1}{2} \tilde{f}_{a}^{b c}\left(\mu_{G}^{(1)}\right)_{b}\left(\mu_{G}^{(1)}\right)_{c}
$$

\footnotetext{
${ }^{2}$ Notice the difference with the negatively graded $T^{*}[-1] N$ of section 4.1 in which one focuses on degree zero charges $S_{0}$ instead of degree two charges (i.e. bivectors) as in the case of $T^{*}[1] N$.
} 
where $\tilde{f}_{a}^{b c}$ are the dual structure constants for $\mathfrak{g}^{*}$. Let us consider the degree 1 reduction data $\left(T^{*}[1] M, \omega=d x^{i} d b_{i}, C_{1}=\mu_{G}^{(1)-1}(0), \pi_{M_{0}}\right)$. If the $G$-action is free and proper then the reduced manifold is

$$
T^{*}[1] M / / C_{1}=T^{*}[1](M / G) \quad .
$$

From eq. (5.19), it follows that $\pi_{M}$ is reducible to a bivector $\pi_{M / G}$ on $M / G$.

The $B F V_{1}$ construction applied to the above reduction data gives

$$
\mathcal{M}:=T^{*}[1] M \times T^{*}[1] \mathfrak{g}[1]
$$

with the symplectic form $\tilde{\omega}=\omega \oplus \varpi$ where $\varpi$ is the canonical degree 1 symplectic structure on $T^{*}[1] \mathfrak{g}[1]=\mathfrak{g}[1] \times \mathfrak{g}^{*}[0]=T^{*}[1] \mathfrak{g}^{*}$. Let us denote with $\left(\xi^{a}, p_{a}\right)$ ghosts and antighosts respectively; the $B F V_{1}$ charge starts with

$$
\Theta_{\pi_{M}}=\pi_{M}+\left(\mu_{G}^{(1)}\right)_{a} \xi^{a}-\frac{1}{2} f_{b c}^{a} p_{a} \xi^{b} \xi^{c}+\ldots \in C^{2}(\mathcal{M})
$$

When $\pi_{M}$ is directly invariant (case $\tilde{f}_{a}^{b c}=0$ ), no more terms are needed above. In the general case, though, due to Poisson-Lie invariance of $\pi_{M}$, corrections are needed in order to satisfy the master equation. If the constraints are regular, from Proposition 11, such a charge exists at least as a formal power series on the antighosts $p_{a}$. We are going to show that it always exists. Let $e: \mathfrak{g}^{*} \rightarrow G^{*}$ be a local diffeomorphism around 0 , such that $e(0)=e \in G$; then one has the lift $\tilde{e}: T^{*}[1] \mathfrak{g}^{*} \rightarrow T^{*}[1] G^{*}$; it is defined on the coordinates $x^{\alpha}, \beta_{\alpha}$ of $T^{*}[1] G^{*}$ as

$$
\tilde{e}^{*}\left(x^{\alpha}\right)=e^{\alpha}(p), \quad \tilde{e}^{*}\left(\beta_{\alpha}\right)=\frac{\partial e_{a}^{-1}}{\partial x^{\alpha}} \xi^{a} .
$$

We assume that $x^{\alpha}$ is zero on the identity $e_{G^{*}} \in G^{*}$. Let us define

$$
\Theta_{\pi_{M}}=\tilde{e}^{*}\left(\pi_{M \triangleleft G^{*}}\right)
$$

where $\pi_{M \triangleleft G^{*}}=\pi_{M}+\pi_{G^{*}}+\left(\mu_{G}^{(1)}\right)_{a} k^{a}$ is the semidirect Poisson structure on $M \times G^{*}$, (see B.36 for notations).

Proposition $21 \Theta_{\pi_{M}}$ is a $B F V_{1}$ charge for the reduction data $\left(T^{*}[1] M, \omega_{1}, \mu_{G}^{(1)}=0, \pi_{M}\right)$.

Proof. Since $\tilde{e}$ is a symplectomorphism, $\Theta_{\pi_{M}}$ is homological. Let us check that $\Theta_{\pi_{M}}$ is negatively ga-graded and has the expansion of eq. (5.21). Keeping in mind the dependence on $\xi$ of (15.22) and that $\pi_{G^{*}}(0)=0$, we easily get that $\Theta_{\pi_{M}}=\sum_{r \leq 1} \Theta_{r}$, where $\Theta_{r}$ is the component of ga-degree $r$. Moreover, we compute that $\Theta_{1}=\left(\mu_{G}^{(1)}\right)_{a} \tilde{\xi}^{a}+\frac{1}{2} f_{a b}^{c} \tilde{\xi}^{a} \tilde{\xi}^{b} \tilde{p}_{c}$ and $\Theta_{0}=\pi_{M}+\tilde{f}_{b}^{a c}\left(\mu_{G}^{(1)}\right)_{a} \tilde{p}_{c} \tilde{\xi}^{b}+O\left(p^{2}\right)$, where $\tilde{\xi}^{a}=k^{a \alpha} e_{b \alpha} \xi^{b}$ and $\tilde{p}_{c}=k_{c \gamma} e^{\gamma d} p_{d}$ defines a symplectomorphism. 
In general $e$ can be chosen as exp, the exponential map, which is in general just a local diffeomorphism around the identity. The construction still works if we restrict ourselves to this neighbourhood of the identity, since this is the region that is relevant for BFVcohomology. Recall also that when $G=K$ is compact and simple then $G^{*}$ is globally diffeomorphic to $\mathfrak{g}^{*}$ (the $A N$ part of the Iwasawa decomposition of $K^{\mathbb{C}}$ ), see [17].

For AKSZ applications of the next sections, we will use this non-linear version of the BFV model, namely, we shall work with the space $\exp \left(B F V_{1}^{\theta}\right):=T^{*}[1]\left(M \times G^{*}\right)$ endowed with the $B F V$ charge $\pi_{M \triangleleft G^{*}}$.

Remark 22 One way of avoiding these "non-linearities", namely the extra terms in the modified charge or the non flat $G^{*}$ factor in the exponentiated version, could be to go one degree higher to $n=2$. In this case, one can encode the Poisson-Lie action information in a Dirac structure [8] inside a Courant algebroid as it will be done in subsection [6.2.

As a final example, let us consider also a momentum map in degree 0 for the $G$-action. Let $G$ act on $\left(M, \pi_{M}\right)$ by Poisson diffeomorphisms, i.e. the Poisson tensor $\pi_{M}$ is $G$ invariant (case $\tilde{f}_{c}^{a b}=0$ ) and assume that this action is hamiltonian. This means that there is a (degree 0) equivariant moment map $\mu_{G}^{(0)}: M \longrightarrow \mathfrak{g}^{*}$ such that the fundamental vector fields are hamiltonian: $v_{a}=\pi_{M}\left(d\left(\mu_{G}^{(0)}\right)_{a}\right)$. We shall consider the constraints defined by the momentum maps $\mu_{G}^{(0)}$ and $\mu_{G}^{(1)}$ altogether, by taking $C_{1}=\left\{\mu_{G}^{(0)}=0, \mu_{G}^{(1)}=0\right\} \subset T^{*}[1] M$. In the regular case, the corresponding reduction yields

$$
T^{*}[1] M / / C_{1}=T^{*}[1]\left(\mu_{G}^{(0)^{-1}}(0) / G\right),
$$

whose functions are the multivectors on the degree 0 reduced space $\mu_{G}^{(0)^{-1}}(0) / G$. The $B F V_{1}$ construction gives

$$
\mathcal{M}=T^{*}[1]\left(M \times \mathfrak{g}[1] \times \mathfrak{g}^{*}[-1]\right)
$$

with $B F V_{1}$ charge given by

$$
\Theta=\Theta_{0}+\Theta_{1}
$$

where

$$
\Theta_{0}=\pi_{M}+p_{a}^{(1)} \xi_{(0)}^{a}, \quad \Theta_{1}=\left(\mu_{G}^{(0)}\right)_{a} \xi_{(0)}^{a}+\left(\mu_{G}^{(1)}\right)_{a} \xi_{(1)}^{a}+\frac{1}{2} p_{c}^{(1)} f_{a b}^{c} \xi_{(1)}^{a} \xi_{(1)}^{b}+f_{b c}^{a} p_{a}^{(0)} \xi_{(0)}^{b} \xi_{(1)}^{c}
$$

and $x^{i}, b_{i}$ are coordinates on $T^{*}[1] M, \xi_{(1)}^{a} \in C^{1}(\mathfrak{g}[1]), \xi_{(0)}^{a} \in C^{2}(\mathfrak{g}[2])$ are the ghosts and $p_{a}^{(0)} \in C^{-1}\left(\mathfrak{g}^{*}[-1]\right), p_{a}^{(1)} \in C^{0}\left(\mathfrak{g}^{*}[0]\right)$ are the corresponding $T^{*}[1]$ conjugates. 
Remark 23 The BFV-AKSZ model corresponding to (5.23) is the sigma model proposed by Zucchini in [35, 36] as Poisson-Weil sigma models and by Signori in [33] under the name of JPSM. In [36] it is shown that a sector of the underlying BV-cohomology is related to the equivariant Poisson cohomology of $\left(M, \pi_{M}\right)$. In Appendix $D$ we clarify this relation, by relating the cohomology of the target QP-manifold to Poisson equivariant cohomology.

\subsection{General reduction for PSM}

We described in Section 5.1 the $B F V_{1}$ construction associated to the Poisson quotient $\left(M, \pi_{M}\right) \rightarrow\left(M / G, \pi_{M / G}\right)$, where $\left(G, \pi_{G}\right)$ is a Poisson Lie group acting on $\left(M, \pi_{M}\right)$. We study here the corresponding BFV-AKSZ models.

Let $\left(G^{*}, \pi_{G^{*}}\right)$ be the dual Poisson-Lie group and recall the semidirect Poisson structure $\pi_{M \triangleleft G^{*}}$ on $M \times G^{*}$. According to what we discussed in Section 5.1, the graded manifold $T^{*}[1]\left(M \times G^{*}\right)$ and hamiltonian $\Theta=\pi_{M \triangleleft G^{*}}$ is a nonlinear version of the $B F V_{1}$ space for these reduction data. The BFV-AKSZ model associated to these data is the PSM of this semidirect structure.

In this section, we shall consider more elaborate gauge fixings than in the general discussion of Sect. 4.2 which make use of the underlying Poisson-Lie geometry. We shall then argue that this PSM with target $\left(M \times G^{*}, \pi_{M \triangleleft G^{*}}\right)$ can be used to compute correlators of PSMs with target $M / H$ for any coisotropic subgroup $H$ of $G$. See the Appendix B for notations and basic results concerning Poisson reduction.

The formal argument goes as follows. First notice that the space of superfields is the direct product of $B V$ manifolds $\mathfrak{F}=\operatorname{Map}\left(T[1] \Sigma, T^{*}[1] M\right) \times \operatorname{Map}\left(T[1] \Sigma, T^{*}[1] G^{*}\right)$. We can then consider the superfields $\Lambda$ with values in $G^{*}$ as ultraviolet degrees of freedom and those $\Phi$ with value in $M$ as infrared. We choose as ultraviolet gauge fixing $\mathcal{L}_{g h}=\operatorname{Map}\left(T[1] \Sigma, N^{*}[1] H^{\perp}\right)$, where $H^{\perp}$ is the connected subgroup (that we assume closed) integrating the annihilator subalgebra $\mathfrak{h}^{\perp} \subset \mathfrak{g}^{*}$ coming from the coisotropic $H \subset G$. Notice that when $H=G$ then $G^{\perp}=\{e\}$ and $N^{*}[1]\{e\}=T_{e}^{*}[1] G^{*}=g[1]$. Thus, in this particular case, the above gauge fixing corresponds to put $p_{a}=0$ in the correspondence (5.22), and so to the ghost gauge fixing discussed in Section 4.2.

The effective action thus yields

$$
e^{\frac{i}{\hbar} S_{\mathrm{eff}}}=\int_{\mathcal{L}_{g h}} D \Lambda e^{\frac{i}{\hbar} S_{\pi_{M}}} G^{*}=e^{\frac{i}{\hbar} S_{\pi_{M}}} \delta_{H},
$$


where

$$
\delta_{H}=\int_{\mathcal{L}} D \Lambda e^{\frac{i}{\hbar} S_{\pi_{G^{*}}}+\frac{i}{\hbar} \int_{T[1] \Sigma}\left(\mu_{G}^{(1)}\right)_{a}(\Phi) k^{a}(\Lambda)}=\int_{\mathcal{L}} D \Lambda e^{\frac{i}{\hbar} \int_{T[1] \Sigma}\left(\mu_{H}^{(1)}\right)_{A}(\Phi) k^{A}(\Lambda)}
$$

is the delta function enforcing the constraint $\mu_{H}^{(1)} \circ \Phi=0$. In the above derivation we used the fact that $\left.\pi_{G^{*}}\right|_{N^{*}[1] H^{\perp}}=0$ due to coisotropy of $H^{\perp},\left\{T_{A}\right\}$ is a basis for $\mathfrak{h}$ and $\left.\left(\mu_{G}^{(1)}\right)_{a} k^{a}\right|_{N^{*}[1] H^{\perp}}=\left(\mu_{H}^{(1)}\right)_{A} k^{A}$ since $H^{\perp}$ is a subgroup. The effective action is then $S_{\pi_{M}}$ restricted to $\mu_{H}^{(1)} \circ \Phi=0$ which, due to the Poisson-Lie invariance and using remark 19, it is equivalent to the reduced action $S_{\text {red }}=S_{\pi_{M / H}}$ on $\mathfrak{F}_{\text {red }}$.

This argument can be made precise in a finite dimensional setting. We will do it by analysing the reduction on the $B V$-theory of zero modes in the next subsection. This analysis must be seen both as a more precise statement of the above argument and as check of the full conjecture.

\subsection{Reduction for zero modes of PSM}

We analyze here the zero modes of the PSM with target the Poisson manifold $(M \times$ $G^{*}, \pi_{M \triangleleft G^{*}}$ ) and source $\Sigma_{g}$, the compact surface of genus $g$. We know from [7] that the zero modes of the PSM with target $\left(M, \pi_{M}\right)$ are described by an AKSZ construction, whose BV-space of superfields is $\mathfrak{F}_{0}^{Z}:=\operatorname{Maps}\left(X_{\Sigma_{g}}, T^{*}[1] M\right)$, where $X_{\Sigma_{g}}$ is the cohomology ring of $\Sigma_{g}$ seen as as sheaf over a point.

We introduce a symplectic basis $\left\{e^{I}, e_{I}\right\}_{I}^{g}$ of $H^{1}\left(\Sigma_{g}\right)$ with the ring structure given by $e_{I} \wedge e^{J}=\delta_{I}^{J} s_{2}$, where $s_{2}$ is the volume form normalized to $\int_{\Sigma_{g}} s_{2}=1$.

Let $\Phi=(\underline{x}, \underline{\eta})$ be the superfields of $\mathfrak{F}_{0}^{Z}$. We then define the genus $g$ momentum map

$$
\zeta_{G, a}^{(g)}=e v^{*}\left(\mu_{G}^{(1)}\right)_{a}=v_{a}(\underline{x})^{i} \underline{\eta}_{i}=\zeta_{a}^{(0)}+\zeta_{a}^{(I)} e_{I}+\zeta_{(I), a} e^{I}+\zeta_{a}^{(2)} s_{2}
$$

The hamiltonian vector fields of the components of $\zeta_{G}^{(g)}$ define an action of a graded Lie algebra $\mathfrak{g}^{(g)}$. The even part is $\mathfrak{g}_{\text {even }}^{(g)}=\left\langle v_{a}^{(2)}\right\rangle \oplus\left\langle v_{a}^{(0)}\right\rangle=\mathfrak{g} \ltimes \mathbb{R}^{\operatorname{dim} \mathfrak{g}}[2]$, where $\mathfrak{g}$ acts on $\mathbb{R}^{\operatorname{dim} \mathfrak{g}}$ with the adjoint action; the odd part is $\mathfrak{g}_{\text {odd }}^{(g)}=\left\langle v_{a}^{(I)}, v_{a(I)}\right\rangle=\mathbb{R}^{2 g}[1]$, where $\mathfrak{g}$ acts on each copy of $\mathbb{R} \subset \mathfrak{g}_{\text {odd }}^{(g)}$ with the adjoint action. The definition of $\zeta_{H}^{(g)}$ and $\mathfrak{h}^{(g)}$ for any subgroup $H \subset G$ is obvious.

Formula (4.15) within this setting means that the space of zero modes of the PSM with target $\left(M / H, \pi_{M / H}\right)$ is obtained as Marsden-Weinstein reduction of $\mathfrak{F}_{0}^{Z}$. One can prove it directly by introducing coordinates of $M$ adapted to the $H$-action:

\section{Lemma 24}

$$
\mathfrak{F}_{\text {red }}^{Z}:=\operatorname{Map}\left(X_{\Sigma_{g}}, T^{*}[1](M / H)\right)=\left(\zeta_{H}\right)^{-1}(0) / \mathfrak{h}^{(g)}
$$


Let us now consider the zero modes of PSM with target the semidirect product Poisson structure on $M \times G^{*}$. Let $\Psi=(\Phi, \Lambda)$ be the superfields of $\mathfrak{F}^{Z}:=\operatorname{Maps}\left(X_{\Sigma_{g}}, T^{*}[1](M \times\right.$ $\left.\left.G^{*}\right)\right)$. The BV-action of zero modes is

$$
S_{\pi_{M \triangleleft G^{*}}}=\int d s_{2} e v^{*} \pi_{M \times G^{*}}=\int d s_{2}\left(\pi_{M}(\Phi)+\pi_{G^{*}}(\Lambda)+v_{a}(\Phi) k^{a}(\Lambda)\right) .
$$

We want to consider $\Lambda$ as UV-degrees of freedom and take $\mathcal{L}_{U V}=\operatorname{Map}\left(X_{\Sigma_{g}}, N^{*}[1] H^{\perp}\right)$ as UV-gauge fixing. In what follows, we shall describe the relevant BV-structure on the space of zero modes.

Let us introduce a volume form $V_{M}=\rho_{M} D x$ on $M$ and a volume form $V_{G^{*}}=\rho_{G^{*}} D \lambda$ on $G^{*}$; let $\nu_{M}=V_{M} \otimes V_{M}$ and $\nu_{G^{*}}=V_{G^{*}} \otimes V_{G^{*}}$ be the corresponding berezinian integration on $T^{*}[1] M$ and $T^{*}[1] G^{*}$, respectively, as in Section 2.2. We denote with $\Delta_{\nu_{M}}$, $\Delta_{\nu_{G^{*}}}$ and $\Delta_{\nu_{M \times G^{*}}}=\Delta_{\nu_{M}}+\Delta_{\nu_{G^{*}}}$ the corresponding $B V$-laplacians. The choice of the volume forms on $M$ and $G^{*}$ define the berezinians $\nu_{M \times G^{*}}^{(g)}$ on the corresponding spaces of zero modes $\mathfrak{F}^{Z}$ as well (see [7] for details). Let us denote with $\Delta_{\nu_{M \times G^{*}}}^{(g)}$ the corresponding $B V$-laplacian and recall the following formula valid for any $F \in C^{\infty}\left(T^{*}[1]\left(M \times G^{*}\right)\right)$

$$
\Delta_{\nu_{M \times G^{*}}}^{(g)} O_{F}=2 s_{2}(1-g) O_{\Delta_{\nu_{M \times G^{*}} F}}
$$

where $O_{F}=\int d s F(\Psi)$. As a consequence, the obstructions for $S_{\pi_{M \triangleleft G^{*}}}$ to solve the quantum master equation are the same as on the target space, namely, that $\pi_{M \triangleleft G^{*}}$ satisfies

$$
\Delta_{\nu_{M \times G^{*}}} e^{\pi_{M \triangleleft G^{*}}}=0 .
$$

In turn, the above equation on target space means that $\pi_{M \triangleleft G^{*}}$ is a unimodular Poisson structure. This is equivalent to

$$
\Delta_{\nu_{G^{*}}} \pi_{G^{*}}+\left(\Delta_{\nu_{M}}\left(\mu_{G}^{(1)}\right)_{a}\right) k^{a}=0, \quad \Delta_{\nu_{M}} \pi_{M}-\left(\Delta_{\nu_{G^{*}}} k^{a}\right)\left(\mu_{G}^{(1)}\right)_{a}=0 \quad .
$$

By using the Poisson Lie property (B.35) for $\pi_{G^{*}}$ and the fact that $\pi_{G^{*}}(e)=0$ we get from the first equation in (15.27) that $\Delta_{\nu_{M}}\left(\mu_{G}^{(1)}\right)_{a}+f_{a b}^{b}=0$. Thus, in particular, there is an induced volume form $V_{M / G}$ on the quotient $M / G$ as in Section 4.1. As a consequence, in the case $H=G$, there is an induced volume $\nu_{M / G}^{(g)}$ on the reduced zero modes field space $\mathfrak{F}_{\text {red }}^{Z}=\operatorname{Map}\left(X_{\Sigma_{g}}, T^{*}[1](M / G)\right)$. Notice that, for more general $H \subset G$, one has to impose additional compatibility conditions to have an induced volume on reduced space $M / H$, namely to ensure that $\Delta_{\nu_{M}}\left(\mu_{G}^{(1)}\right)_{A}+f_{A B}^{B}=0$. Observe that in the case $G$ is compact semisimple and $H \subset G$ closed, one always obtain the desired induced volume on $M / H$.

Remark 25 Equation (5.27) implies that $\Delta_{\nu_{M}}\left(\mu_{G}^{(1)}\right)_{a}=\operatorname{div}_{V_{M}} v_{a}=-c_{a} \in \mathbb{R}, \Delta_{\nu_{G^{*}}} \pi_{G^{*}}=$ $c_{a} k^{a}, \Delta_{\nu_{G^{*}}} k^{a}=\operatorname{div}_{V_{G^{*}}} k^{a}=-c^{a} \in \mathbb{R}$ and $\Delta_{\nu_{M}} \pi_{M}=c^{a}\left(\mu_{G}^{(1)}\right)_{a}$. Recall that if a vector field has constant non zero divergence then the volume form is exact; for instance if $M$ is compact then $c_{a}=0$, i.e. $v_{a}$ is divergenceless, and $\pi_{G^{*}}$ is unimodular. 
The effective action gives

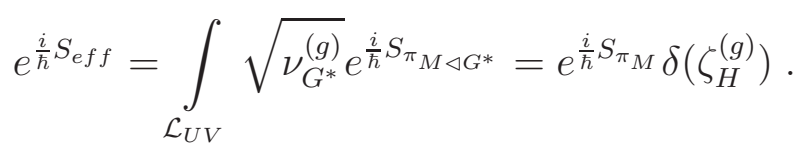

From the discussion in remarks 19 the above effective action can be considered to be equivalent to the reduced zero-modes action $S_{\pi_{M / H}}$. Also above, notice that for $g \neq 0$ the momentum map $\zeta_{H}^{(g)}$ also contains even components so that the delta function must be considered as an ordinary distribution 3 .

Let us now focus on the case $H=G$ and discuss the gauge fixing of the infrared degrees of freedom.

Let $f \in C^{\infty}\left(T^{*}[1] M\right)$ be reducible, i.e. it satisfies

$$
\left\{\left(\mu_{G}^{(1)}\right)_{a}, f\right\}=f_{a}^{b}\left(\mu_{G}^{(1)}\right)_{b}, \quad\left\{\pi_{M}, f\right\}=f^{b}\left(\mu_{G}^{(1)}\right)_{b},
$$

so that it induces an $f_{\text {red }} \in C^{\infty}\left(T^{*}[1] M / G\right)$ on the quotient $q: M \rightarrow M / G$. Suppose we have $F \in C^{\infty}\left(T^{*}[1]\left(M \times G^{*}\right)\right)$ a corresponding extended observable satisfying $\left.F\right|_{T^{*}[1] M}=f$ and $\Delta_{\nu_{M \times G^{*}}} F=0$. Let $\tilde{\Gamma} \subset M / G, \Gamma \subset M$ as in Lemma 15 ,

On the full zero modes field space now, as a consequence of (5.26), we get that $O_{F}$ is a quantum observable and let us consider as IR-gauge fixing the lagrangian submanifold $\mathcal{L}_{I R}=\operatorname{Map}\left(X_{\Sigma_{g}}, N^{*}[1] \Gamma\right)$. As in section 4.1, we obtain the following characterization of correlators.

Proposition 26 With the notations above, the correlator yields

$$
\begin{aligned}
\left\langle O_{F}\right\rangle_{\nu_{M}}=\int_{\mathcal{L}_{U V} \times \mathcal{L}_{I R}} \sqrt{\nu_{M}^{(g)} \nu_{G^{*}}^{(g)}} O_{F} e^{\frac{i}{\hbar} S_{\pi_{M \triangleleft G}}} & =\int_{\mathcal{L}_{I R}} \sqrt{\nu_{M}^{(g)}} O_{f} e^{\frac{i}{\hbar} S_{\pi_{M}}} \delta\left(\zeta_{G}^{(g)}\right) \\
& =\int_{\left\{X_{\Sigma} \rightarrow N^{*}[1] \tilde{\Gamma}\right\}} \sqrt{\nu_{M / G}^{(g)}} O_{f_{r e d}} e^{\frac{i}{\hbar} S_{\pi_{M / G}}} \\
& =\left\langle O_{f_{\text {red }}}\right\rangle_{\nu_{M / G}} .
\end{aligned}
$$

It thus depends only on $f_{\text {red }}$ and on the homology class of $\tilde{\Gamma}$.

The proof can be done by considering a cover of adapted coordinates on the principal bundle $M \rightarrow M / G$.

Notice that the transversality condition on $\Gamma$ with respect to the quotient $q: M \rightarrow$ $M / G$ is also a necessary condition for the above integral be different from zero, since $\left.\zeta_{G}^{(g)}\right|_{\mathcal{L}_{I R}}=\left.e v^{*} \mu_{G}^{(1)}\right|_{N^{*}[1] \Gamma}$.

\footnotetext{
${ }^{3}$ Notice that to get these even delta functions the $i$ factor on the integrand $e^{\frac{i}{\hbar} S}$ becomes important.
} 


\section{Reduction of a group action for $n=2$}

We give in this Section the details for the $B F V_{2}$ construction in the case of a group action. Symplectic NQ manifolds of degree 2 encode the structure of Courant algebroid, so that the geometric background is about the reduction of Courant algebroids. The main reference for this topic is [9]; we sketch some facts in Appendix C. We shall consider in particular the case of exact Courant algebroids, i.e. the reduction by a group action of the $n=2$ symplectic manifold $M_{2}=T^{*}[2] T^{*}[1] M_{0}$.

Let $G$ act freely on $M$ with $v_{a}=v_{a}^{i} \partial_{i} \in \mathfrak{X}(M)$ being the infinitesimal generators. The $G$-action on $M$ lifts naturally to $T^{*}[1] M$ and this one, in turn, lifts naturally to the symplectic $\mathcal{M}_{0}=T^{*}[2] T^{*}[1] M$. This action is hamiltonian with moment map

$$
\begin{aligned}
\mu_{G}^{(2)} & : \quad T^{*}[2] T^{*}[1] M \longrightarrow \mathfrak{g}^{*}[2] \\
\left(\mu_{G}^{(2)}\right)_{a} & =v_{a}^{i} p_{x^{i}}-\partial_{j} v_{a}^{k} b_{k} p_{b_{j}}
\end{aligned}
$$

where $\left(x^{i}, b_{j}\right)$ are graded coordinates on $T^{*}[1] M$ and $\left(p_{x^{i}}, p_{b_{j}}\right)$ denote the corresponding conjugated variables on $T^{*}[2] T^{*}[1] M$, of degree 2 and 1 respectively, with respect to the canonical degree 2 symplectic form $\omega_{2}$. We will also consider the degree one moment map

$$
\mu_{G}^{(1)}: T^{*}[2] T^{*}[1] M \rightarrow \mathfrak{g}^{*}[1] \quad, \quad\left(\mu_{G}^{(1)}\right)_{a}=v_{a}^{i} b_{i} ;
$$

the collection of moment maps $\mu_{G}=\left(\mu_{G}^{(2)}, \mu_{G}^{(1)}\right)$ satisfies the algebra (C.38), (for $\lambda=0$ ). We consider the coisotropic submanifold $\mathcal{C}=\mu_{G}^{-1}(0) \hookrightarrow T^{*}[2] T^{*}[1] M$; the symplectic reduction of these constraints gives

$$
\mathcal{M}_{0} / / \mathcal{C}=T^{*}[2] T^{*}[1](M / G) .
$$

Following the general procedure of Section (3.1), we get for the $B F V_{2}$ space

$$
\mathcal{M}=T^{*}[2]\left(T^{*}[1] M \times \mathfrak{g}[1] \times \mathfrak{g}[2]\right) .
$$

Let us denote with $\xi_{(2)}\left(\right.$ resp. $\left.\xi_{(1)}\right)$ the ghosts associated to $\mu_{G}^{(2)}\left(\right.$ resp. $\left.\mu_{G}^{(1)}\right)$, and $p_{\xi^{(2)}}$ $\left(\right.$ resp. $\left.p_{\xi^{(1)}}\right)$ the momenta, we see by a direct computation that the $B F V_{2}$ charge is

$$
\Theta_{1}=\left(\mu_{G}^{(2)}\right)_{a} \xi_{(2)}^{a}+\left(\mu_{G}^{(1)}\right)_{a} \xi_{(1)}^{a}-\frac{1}{2} f_{a b}^{c} \xi_{(2)}^{a} \xi_{(2)}^{b} p_{\xi_{(2)}^{c}}-f_{a b}^{c} \xi_{(2)}^{a} \xi_{(1)}^{b} p_{\xi_{(1)}^{c}} .
$$

Observe that $\mathcal{M}$ is nonnegatively graded so that the $B F V_{2}$ hamiltonian (6.30) defines a Courant algebroid structure on $\left(T+T^{*}\right)\left(M \times \mathfrak{g}^{*}\right)$. 


\subsection{Reduction of an exact Courant algebroid}

We want now to encode the reduction of exact Courant algebroids, as described in Appendix C. Let us consider the exact Courant algebroid defined by the hamiltonian $\theta_{H} \in$ $C^{3}\left(T^{*}[2] T^{*}[1] M\right)$, where $\theta_{H}=p_{b_{i}} p_{x^{i}}-\frac{1}{6} H_{i j k} p_{b_{i}} p_{b_{j}} p_{b_{k}}$, with $H$ being the closed three-form representing the Severa class. We suppose that the $G$-action is isotropic and trivially extended so that there exist one forms $\lambda_{a}=\lambda_{a, i} p_{b_{i}}$ such that $\Phi=H+\sum_{a} t_{a} \lambda_{a}$ is equivariantly closed. We slightly modify the above construction just by redifining the degree one constraints to

$$
\begin{aligned}
\mu_{G \lambda}^{(1)} & : \quad T^{*}[2] T^{*}[1] M \rightarrow \mathfrak{g}^{*}[1] \\
\left(\mu_{G \lambda}^{(1)}\right)_{a} & =v_{a}^{i} b_{i}+\lambda_{a, i} p_{b_{i}} .
\end{aligned}
$$

The constraints $\mu_{G}=\left(\mu_{G}^{(2)}, \mu_{G \lambda}^{(1)}\right)$ still close the algebra (C.38) (see discussion in the Appendix (C) and $\theta_{H}$ is invariant under the action of the corresponding hamiltonian vector fields. The symplectic reduction still gives $\mathcal{M}_{0} / / \mathcal{C}=T^{*}[2] T^{*}[1](M / G)$ and the hamiltonian $\theta_{H}$ descends to $\theta_{H}^{\text {red }}$ that defines the quotient Courant algebroid. The $B F V_{2}$ charge is easily seen to be

$$
\Theta_{H}=\theta_{H}+\Theta_{1}
$$

where $\Theta_{1}$ is the same in (6.30) provided on takes $\mu_{G \lambda}^{(1)}$ as degree one constraints. This is the BFV-model for the Courant algebroid reduction. Finally, the new hamiltonian $\Theta_{H}$ defines another Courant algebroid structure on the pseudo euclidean vector bundle $E=\left(T+T^{*}\right)\left(M \times \mathfrak{g}^{*}\right)$.

\subsection{Courant algebroid related to a Poisson action}

We can further modify the above construction by taking into account the subalgebra of the algebra of constraints given by those of degree two, i.e. let us consider the coisotropic $\mathcal{C}^{\prime}=\mu_{G}^{(2)-1}(0) \subset \mathcal{M}_{0}$. In this case, $\mathcal{M}_{0} / / \mathcal{C}^{\prime}=T^{*}[2]\left(\frac{T^{*}[1] M}{G}\right)$ and the $B F V_{2}$ construction yields

$$
\mathcal{M}^{\prime}=T^{*}[2]\left(T^{*}[1] M \times \mathfrak{g}[1]\right)
$$

endowed with canonical degree 2 symplectic structure $\omega_{2}^{\prime}$ and with the $B F V_{2}$ charge

$$
\Theta_{1}^{\prime}=\left(\mu_{G}^{(2)}\right)_{a} \xi^{a}+\frac{1}{2} f_{b c}^{a} p_{\xi^{a}} \xi^{b} \xi^{c}
$$

where $\xi^{a} \in C^{1}(\mathfrak{g}[1])$ and $p_{\xi^{a}} \in C^{1}\left(\mathfrak{g}^{*}[1]\right)$ are conjugate coordinates on $T^{*}[2](\mathfrak{g}[1])$.

Now we want to bring a Poisson structure $\pi=\pi^{i j} b_{i} b_{j}$ on $M$ into the picture. To that end, let us consider the hamiltonian $\theta_{\pi} \in C^{3}\left(T^{*}[2] T^{*}[1] M\right)$

$$
\theta_{\pi}=p_{b_{j}} p_{x^{j}}+p_{x^{i}} \pi^{i j} b_{j}+\frac{1}{2} p_{b_{i}} \partial_{i} \pi^{k l} b_{k} b_{l}
$$


satisfying

$$
\left\{\theta_{\pi}, \theta_{\pi}\right\}=0
$$

The hamiltonian $\theta_{\pi}$ corresponds to the Courant algebroid structure on $T^{*} M \oplus T M$ obtained as the double of the Lie bialgebroid $\left(T_{\pi}^{*} M, T M\right)$ [24, which in turn is isomorphic (though not equal) to the standard Courant algebroid structure (see also the Lemma below).

If the $G$ action is Poisson, i.e. it satisfies (5.19), then one can check that $\theta_{\pi}$ is reducible, i.e. it satisfies

$$
\left\{\theta_{\pi},\left(\mu_{G}^{(2)}\right)_{a}\right\}=\tilde{f}_{a}^{b c}\left(\mu_{G}^{(1)}\right)_{b}\left(\mu_{G}^{(2)}\right)_{c}
$$

By direct check we obtain the following characterization of the $B F V_{2}$ charge.

Lemma 27 The $B F V_{2}$ charge is given by

$$
\Theta_{\pi}=\Theta_{\pi 0}+\Theta_{\pi 1}=\theta_{\pi}+\left(\mu_{G}^{(2)}\right)_{a} \xi^{a}+\frac{1}{2} f_{b c}^{a} p_{\xi^{a}} \xi^{b} \xi^{c}-\tilde{f}_{a}^{b c}\left(\mu_{G}^{(1)}\right)_{b} p_{\xi^{c}} \xi^{a}-\frac{1}{2} \tilde{f}_{a}^{b c} p_{\xi^{b}} p_{\xi^{c}} \xi^{a}
$$

The symplectomorphism

$$
\phi_{\pi}: T^{*}[2]\left(T^{*}[1] M \times \mathfrak{g}[1]\right) \longrightarrow T^{*}[2]\left(T^{*}[1] M \times \mathfrak{g}[1]\right),
$$

given on coordinate functions by

$$
\begin{aligned}
\phi_{\pi}^{*}\left(p_{b_{i}}\right) & =p_{b_{i}}+\pi^{i j} b_{j}+v_{a}^{i} \xi^{a} \\
\phi_{\pi}^{*}\left(p_{x^{i}}\right) & =p_{x^{i}}+\frac{1}{2} \partial_{i} \pi^{k l} b_{k} b_{l}-\partial_{i} v_{a}^{k} \xi^{a} b_{k} \\
\phi_{\pi}^{*}\left(p_{\xi^{a}}\right) & =p_{\xi^{a}}-v_{a}^{i} b_{i}
\end{aligned}
$$

and the identity on the other coordinates, satisfies

$$
\Theta_{\pi}=\phi_{\pi}^{*}\left(\Theta^{0}\right)
$$

where

$$
\Theta^{0}:=p_{b_{i}} p_{x^{i}}+\frac{1}{2} f_{b c}^{a} p_{\xi^{a}} \xi^{b} \xi^{c}-\frac{1}{2} \tilde{f}_{a}^{b c} p_{\xi^{b}} p_{\xi^{c}} \xi^{a}
$$

is the hamiltonian corresponding to the product Courant algebroid structure of the standard $\left(T^{*} M \oplus T M\right)$ with the double of the bialgebra $\left(\mathfrak{g} \oplus \mathfrak{g}^{*}\right)$.

The main point here is that the submanifold $\mathcal{L}_{\pi} \subset \mathcal{M}^{\prime}$ of the $B F V_{2}$ construction above obtained by setting $\phi^{*}\left(p_{b_{i}}\right)=\phi^{*}\left(p_{x^{i}}\right)=\phi^{*}\left(p_{\xi^{a}}\right)=0$, is lagrangian and corresponds to the Dirac structure of $\left(T^{*} M \oplus T M\right) \times\left(\mathfrak{g} \oplus \mathfrak{g}^{*}\right)$ which encodes the Poisson-Lie action of $G$ on $\left(M_{0}, \pi\right)$ as described in 8 .

Finally, we mention that the associated 3d AKSZ-BFV models will be the Courant sigma models with target the Courant algebroid $\left(T^{*} M \oplus T M\right) \times\left(\mathfrak{g} \oplus \mathfrak{g}^{*}\right)$. The properties of these models with respect to reduction should go along the general lines of Section 4.2, and will be studied elsewhere. 
Remark 28 One could also discuss the corresponding 3d AKSZ-BFV models in which $\Sigma_{3}$ has boundary and the boundary condition for the fields is taken to be the one determined by $\mathcal{L}_{\pi}$.

\section{Summary and outlook}

We have presented a framework to encode systematically the reduction of the target space geometry into ASKZ sigma models. This is provided by the construction of the $B F V-A S K Z$ model where one has a clear picture of the role of the ingredients involved. We then studied how this model is related to the underlying reduced one, showing that the correlators of the reduced theory can be formally obtained from the $B F V-A S K Z$ model by imposing suitable gauge fixings. Moreover, we implemented our construction in particular cases and showed that we recover several of the gauged models that were previously considered in the literature, thus providing a clear conceptual context for their study.

Notice, however, that the framework that we discussed is very general and can be applied to all kind of symplectic reduction in graded symplectic geometry. So, let us now discuss some possible future directions.

Consider a Poisson manifold obtained as a quotient of a symplectic one; in this case the $B F V-A K S Z$ theory will give an extension of the PSM with target a symplectic manifold by adding ghost and antighosts. In the symplectic case the PSM is equivalent to the Amodel by choosing the gauge fixing given by the choice of an almost complex structure (see [6]); putting together this with the ghost gauge fixing we can think of studying the non perturbative properties of the reduced model, so far unaccessible for the generic Poisson case.

On the other hand, when a Lie group $G$ acts by symmetries on a Poisson manifold, the perturbative quantization of the corresponding $B F V-A K S Z$ model could be related to the $G$-equivariant version of Kontsevich's formality. Something similar could be also explored for the case in which the $G$ action is hamiltonian and one wants to get star products on $\mu_{0}^{-1}(0) / G$.

More generally, take an integrable Poisson manifold with symplectic groupoid $\Gamma(M)$. Then the right invariant vector fields define a coisotropic submanifold of $T^{*}[1] \Gamma$ whose symplectic reduction is $T^{*}[1] M$. The $B F V$ manifold will be a non negatively graded $n=1$ symplectic manifold, i.e. a Poisson manifold containing the symplectig groupoid 
as a submanifold. Of course in this case the problem of explicitly computing the $B F V$ hamiltonian can be rather difficult to be solved in general.

We will come back to these examples in the future.

\section{A Quantization of Hamiltonian systems with symme- try}

Let $\left(T^{*} Q, \omega_{0}, G, J, H \in C^{\infty}\left(T^{*} Q\right)\right)$ be a hamiltonian system with symmetry, where the moment map is $J: T^{*} Q \longrightarrow \mathfrak{g}^{*}$. The underlying first class constrained classical action is

$$
S_{c l}=\int_{I} d t p_{i} \dot{x}^{i}-H(x, p)-\lambda^{a} J_{a}\left(x^{i}, p_{i}\right)=S_{c l}^{0}-\int_{I} d t \lambda^{a} J_{a}\left(x^{i}, p_{i}\right)
$$

where $I=[0, T]$ denotes a time interval. Assuming 0 is a regular value for $J$, the corresponding $B F V_{n=0}$ construction yields $\left(\mathcal{M}:=T^{*} Q \times \mathfrak{g}[1] \times \mathfrak{g}^{*}[-1], \omega_{0} \oplus \varpi_{0}, \Theta_{1}\right)$ as in Section 3.1. The BFV charge is given by

$$
\Theta_{1}\left(x^{i}, p_{i}, \xi^{a}, p_{a}\right)=\xi^{a} J_{a}\left(x^{i}, p_{i}\right)-\frac{1}{2} f_{a b}^{c} p_{c} \xi^{a} \xi^{b}
$$

where $x^{i}, p_{i}$ are canonical coordinates on $T^{*} Q, \xi^{a}$ are $\operatorname{deg} 1$ coordinates on $g[1]$ and $p_{a}$ their deg -1 conjugates.

We shall mimic the BFV-AKSZ procedure of Section 4.2, but adapted to the nontopological case as follows. Consider the space of BV fields and antifields to be

$$
\mathfrak{F}=\operatorname{Map}\{T[1] I, \mathcal{M}\} \simeq T[-1] T^{*} P Q
$$

where $P Q=\{I \rightarrow Q\}$ denotes the path space and the fields are assumed to satisfy appropriate boundary conditions. We take the extended BV action to be

$$
S_{B V}=\int_{T[1] I} \boldsymbol{p}_{x^{i}} d \boldsymbol{x}^{i}+\boldsymbol{p}_{\xi^{a}} d \boldsymbol{\xi}^{a}-\Theta_{1}\left(\boldsymbol{p}_{i}, \boldsymbol{x}^{i}, \boldsymbol{\xi}^{a}, \boldsymbol{p}_{c}\right)-H\left(x, p_{x}\right) \theta_{t} \in C(\mathfrak{F})
$$

Notice that by setting the BV fields with non-zero degree to zero one recovers the classical constrained action $S_{c l}$. The only difference with the usual AKSZ construction is the last term involving $\theta_{t} \equiv d t$ on $T[1] I$. The CME is still satisfied

$$
\left\{S_{B V}, S_{B V}\right\}=0
$$

but it only reflects the $G$-symmetry, the $\operatorname{Diff}(I)$ invariance is broken by the term with $H \neq 0$. This agrees with the fact that the theory is non-topological since it has non-trivial hamiltonian dynamics. 
Let us now consider an appropriate gauge fixing. For simplicity, assume $G$ acts on $Q$ freely and properly and that the action on $T^{*} Q$ is the lifted one. Let us choose $\Gamma \subset Q$ transverse to the $G$-action and set $W:=\left.T^{*} Q\right|_{\Gamma} \subset T^{*} Q$. Notice these are chosen so that $W \cap J^{-1}(0) \simeq T^{*}(Q / G)$ yields the reduced phase space.

Noticing that $P W \subset P T^{*} Q \simeq T^{*} P Q$, we can thus define the Lagrangian gauge fixing to be $\mathfrak{L}:=N^{*}[-1](P W) \subset T^{*}[-1] T^{*} P Q \simeq \mathfrak{F}$.

Remark 29 Let $\tilde{x}^{\alpha}, y^{a}$ denote adapted coordinates for which $Q \simeq Q / G \times G$, and $\tilde{p}_{\alpha}, z_{a}$ their conjugates on $T^{*} Q$. Then, $P W \simeq\left\{y^{a}(t)=0\right\}$ and

$$
\mathfrak{L} \simeq\left\{y^{a}(t)=0, \tilde{x}_{(1)}^{\alpha}(t)=0=\tilde{p}_{\alpha}^{(1)}(t), y_{(1)}^{a}(t)=0\right\}
$$

recalling the notation for the $B V$ fields is $e v^{*} \phi=\phi(t)+\phi_{(1)}(t) \theta_{t}$.

One can then compute the partition function as follows

$$
\begin{aligned}
Z=\int_{\mathfrak{L}} e^{i S_{B V}} & =\int_{P W} e^{\left.i S_{c l}^{0}\right|_{P W}} \int D \xi^{a} D \xi_{(1)}^{a} D z_{a}^{(1)} e^{-i \int_{I} J_{a} \xi_{(1)}^{a}+\xi^{a}\left\{J_{a}, y^{b}\right\} z_{b}^{(1)}} \\
& =\int_{T^{*} P Q} e^{i S_{c l}^{0} \delta\left(J_{a}\right) \delta\left(y^{b}\right) \operatorname{det}\left(\left\{J_{a}, y^{b}\right\}\right)} \\
& =\int_{T^{*} P(Q / G)} e^{i \tilde{S}_{c l}^{0} \operatorname{det}\left(\left.\left\{J_{a}, y^{b}\right\}\right|_{J_{a}=0=y^{b}}\right)=Z_{r e d}}
\end{aligned}
$$

Renaming the degree 0 coordinates $\xi_{(1)}^{a}=: \lambda^{a}$, the above is exactly the expression given in [15] for the path integral on reduced spaces. See also [18] where they also explain the relation to the usual BFV hamiltonian quantization [19].

\section{B Basic facts of Poisson actions}

Let us review here basic facts of Poisson reduction. For a systematic treatment in terms of the supergeometric language see [14].

Let $G$ be a Lie group acting freely and properly on $M$. Let $\left\{x^{i}\right\}$ be coordinates on $M_{0}$ and $\left\{b_{i}\right\}$ the odd coordinates on the fibre of $T^{*}[1] M$. Let us introduce a basis $\left\{T_{a}\right\}$ of $\mathfrak{g} \equiv$ Lie $G$ and let $v_{a}=v_{a}^{i} \partial_{i}$ be the fundamental vector field of $T_{a} \in \mathfrak{g}$ and $\left(\mu_{G}^{(1)}\right)_{a}=v_{a}^{i} b_{i}$ the corresponding degree one momentum map which lifts the $G$ action to an hamiltonian action on $T^{*}[1] M$ so that

$$
T^{*}[1](M / G)=\left(\mu_{G}^{(1)}\right)^{-1}(0) / G
$$

\footnotetext{
${ }^{4}$ One can check that the above expression is indeed independent of the choice of adapted coordinates.
} 
As usual, the above quotient means that $\left(\mu_{G}^{(1)}\right)^{-1}(0)$ is the submanifold of $T^{*}[1] M$ defined by the ideal $I_{\mu_{G}^{(1)}} \subset C^{\infty}\left(T^{*}[1] M\right)$ generated by $\mu_{G}^{(1)}$ and $T^{*}[1](M / G)$ is the graded manifold whose functions are $C^{\infty}\left(\left(\mu_{G}^{(1)}\right)^{-1}(0)\right)^{i n v}$, the invariant functions on $\left(\mu_{G}^{(1)}\right)^{-1}(0)$ with respect to the $G$ action. For any subgroup $H \subset G$ with Lie algebra $\mathfrak{h}$, we denote the moment map with $\mu_{H}^{(1)}=\operatorname{pr}_{h} \circ \mu_{G}^{(1)}$, where $\operatorname{pr}_{h}: \mathfrak{g}^{*} \rightarrow \mathfrak{g}^{*} / \mathfrak{h}^{\perp}=\mathfrak{h}^{*}$.

As in section 5.1, let us assume now that $M$ is a Poisson manifold with tensor $\pi_{M}=$ $\frac{1}{2} \pi^{i j} b_{i} b_{j}, G$ is a Poisson Lie group with tensor $\pi_{G}$ and that the action is Poisson. This means that

$$
\left\{\left(\mu_{G}^{(1)}\right)_{a}, \pi_{M}\right\}=\frac{1}{2} \tilde{f}_{a}^{b c}\left(\mu_{G}^{(1)}\right)_{b}\left(\mu_{G}^{(1)}\right)_{c},
$$

where $\tilde{f}_{a}^{b c}$ are the structure constants of $\mathfrak{g}^{*}$. To these data we can associate the following constructions (see for instance [26]):

i) Poisson reduction. Let $H \subset G$ be a closed subgroup of $G$ which is coisotropic with respect to $\pi_{G}$, i.e. $\pi_{G}\left(N^{*} H\right) \subset T H$. We recall that, then, the annihilator $\mathfrak{h}^{\perp}$ is a subalgebra of $\mathfrak{g}^{*}$; it follows from (B.35) that $\pi_{M} \in C^{2}\left(T^{*}[1] M\right)$ descends to $\pi_{M / H} \in$ $C^{2}\left(T^{*}[1](M / H)\right)$, i.e. on $M / H$ there exists a unique Poisson structure such that the projection $M \rightarrow M / H$ is Poisson.

ii) A Poisson structure on $M \times G^{*}$. Let $G^{*}$ be the dual Poisson Lie group of $G$ with Poisson tensor $\pi_{G^{*}}$. Then we can define on $M \times G^{*}$ a Poisson structure. Let $\left\{\lambda_{a}\right\}$ be

coordinates on $G^{*}$ and $\left\{\beta^{a}\right\}$ on the fibre of $T^{*}[1] G^{*}$. Let us denote with $k^{b}=k_{a}^{b} \frac{\partial}{\partial \lambda_{a}}$ the left invariant vector fields of $G^{*}$. The tensor

$$
\pi_{M \triangleleft G^{*}}=\pi_{M}+\pi_{G^{*}}+v_{b}^{i} k_{a}^{b} b_{i} \beta^{a}=\pi_{M}+\pi_{G^{*}}+\left(\mu_{G}^{(1)}\right)_{b} k^{b}
$$

is Poisson as a consequence of (B.35).

\section{Reduction of Courant algebroids}

The main source for reduction of Courant algebroids is [9. The description in terms of graded manifold language can be found in [10]. Here we will consider the particular situation of a trivially extended isotropic action.

An exact Courant algebroid is given by the following operations on the space of section of $E=T M+T^{*} M$ :

i) the pairing $\langle v+\omega, w+\nu\rangle=\frac{1}{2}(\omega(w)+\nu(v))$;

ii) the Courant bracket $[v+\omega, w+\nu]=[v, w]+\mathcal{L}_{v} \nu-\iota_{w} \omega+\iota_{w} \iota_{v} H$ 
where $v, w \in \mathcal{X}(M), \omega, \nu \in \Omega^{1}(M)$ and $H \in \Omega^{3}(M)$ is a closed three form representing the so called Severa class of $E$. It can be encoded in the graded manifold language as follows. Let us consider the symplectic graded manifold $T^{*}[2] T^{*}[1] M$ of degree 2 . In terms of the local Darboux coordinates $\left(x^{i}, b_{i}, p_{b_{i}}, p_{x^{i}}\right)$ of degree $(0,1,1,2)$, the homological hamiltonian encoding the pairing and the Courant bracket reads

$$
\theta_{H}=p_{x^{i}} p_{b_{i}}+\frac{1}{6} H_{i j k} p_{b_{i}} p_{b_{j}} p_{b_{k}}
$$

Let the Lie group $G$ act on $M$ freely and let $v_{X}$ denote the fundamental vector field of $X \in \mathfrak{g}$. Following [9], a trivial extension of this action to the Courant algebroid $E$ is a map $\rho: \mathfrak{g} \rightarrow \Gamma(E)$ preserving the splitting of $E$ and the Courant bracket. If we denote $\rho(X)=v_{X}+\lambda_{X} \in T M+T^{*} M$ for $X \in \mathfrak{g}$, these two conditions mean that for any $X, Y \in \mathfrak{g}$ we have

$$
v_{[X, Y]}=\left[v_{X}, v_{Y}\right], \quad \iota_{v_{X}} H=d \lambda_{X}, \quad \lambda_{[X, Y]}=\mathcal{L}_{X} \lambda_{Y} .
$$

As a consequence $\Phi=H+\sum_{a} t_{a} X_{a} \in C^{3}\left(T[1] M \times \mathfrak{g}^{*}[2]\right)$ is an equivariant form in the Cartan complex for equivariant cohomology and one computes $d_{G}(\Phi)=\langle\rho(\mathfrak{g}), \rho(\mathfrak{g})\rangle$, where $d_{G}=d+\sum_{a} t_{a} \iota_{v_{a}}$. The action is isotropic if $d_{G}(\Phi)=0$ so that $\Phi$ is an equivariant extension of $H$; we say that $\rho$ defines an isotropic trivial extension of $v$.

The general procedure of reduction described in Proposition 3.6 of [9] gives a reduced Courant algebroid $E_{\text {red }}$ on $M / G$ which is exact. The reduced Severa class is $[\Phi] \in H_{G}^{3}(M, \mathbb{R}) \sim H^{3}(M / G)$.

In terms of graded manifold language, the above setting is described by the constraints $\left(\mu_{G}^{(2)}\right)_{a} \in C^{1}\left(T^{*}[2] T^{*}[1] M\right)$ and $\left(\mu_{G \lambda}^{(1)}\right)_{a} \in C^{2}\left(T^{*}[2] T^{*}[1] M\right)$ defined as

$$
\left(\mu_{G}^{(2)}\right)_{a}=v_{a}^{i} p_{i}-\partial_{i} v_{a}^{j} b_{j} p_{b_{i}}, \quad\left(\mu_{G \lambda}^{(1)}\right)_{a}=v_{a}^{i} b_{i}+\lambda_{a i} p_{b_{i}} .
$$

Since the action is isotropic, they satisfy the algebra

$$
\left\{\left(\mu_{G}^{(2)}\right)_{a},\left(\mu_{G}^{(2)}\right)_{b}\right\}=f_{a b}^{c}\left(\mu_{G}^{(2)}\right)_{c}, \quad\left\{\left(\mu_{G}^{(2)}\right)_{a},\left(\mu_{G \lambda}^{(1)}\right)_{a}\right\}=f_{a b}^{c}\left(\mu_{G \lambda}^{(1)}\right)_{c}, \quad\left\{\left(\mu_{G \lambda}^{(1)}\right)_{a},\left(\mu_{G \lambda}^{(1)}\right)_{a}\right\}=0 .
$$

Moreover, since $H$ is $\mathfrak{g}$-invariant then we have that

$$
\left\{\left(\mu_{G}^{(2)}\right)_{a}, \theta_{H}\right\}=\left\{\left(\mu_{G \lambda}^{(1)}\right)_{a}, \theta_{H}\right\}=0,
$$

showing that $\theta_{H}$ is reducible.

\section{Relation to Poisson equivariant cohomology}

Equivariant Poisson cohomology is defined in [16] for a Poisson Lie $G$-manifold $M$ endowed with a (pre-)momentum map. Here we will concentrate on the case in which 
$\left(G, \pi_{G}=0\right)$ acts hamiltonianly on $\left(M, \pi_{M}\right)$ with equivariant moment map $\mu_{G}^{(0)}: M \rightarrow \mathfrak{g}^{*}$. This corresponds to the case $G^{*}=\mathfrak{g}^{*}$ and the $B F V_{1}$ construction has been discussed at the end of Subsection 5.1.

Let us first say a few words about equivariant Poisson cohomology of $\left(M, \pi_{M}, G, \mu_{G}^{(0)}\right)$ following [16]. One considers a Weil model complex as

$$
A:=\left(\mathfrak{X}(M) \otimes W(\mathfrak{g}), d_{A}=d_{\pi_{M}}+d_{W}\right)
$$

where $W(\mathfrak{g})=S \mathfrak{g}^{*} \otimes \Lambda \mathfrak{g}^{*}$ denotes the Weil algebra of $\mathfrak{g}$ endowed with the usual Weil differential $d_{W}$ and $d_{\pi}=[\pi$,$] denotes Poisson cohomology differential. Next, define the$ subcomplex $A_{b} \subset A$ formed by basic elements $F \in A$ such that

$$
\begin{array}{r}
\left(i_{d\left(\mu_{G}^{(0)}\right)_{a}}+I_{a}^{W}\right) F=0 \\
\left(L_{v_{a}}+L_{a}^{W}\right) F=0
\end{array}
$$

where $i_{d\left(\mu_{G}^{(0)}\right)_{a}}$ denotes contraction and $L_{v_{a}}$ Lie derivative on $\mathfrak{X}(M)$ while $I_{a}^{W}, L_{a}^{W}$ denote the usual contraction and $\mathfrak{g}$-action operations on $W(\mathfrak{g})$. Equivariant Poisson cohomology $H_{G}\left(M, \pi_{M}, \mu_{G}^{(0)}\right)$ is defined as the cohomology of the subcomplex $\left(A_{b},\left.d_{A}\right|_{A_{b}}\right) \subset\left(A, d_{A}\right)$.

The corresponding $B F V_{1}$ space has been studied at the end of subsection 5.1. As a degree one symplectic manifold it is

$$
\mathcal{M}=T^{*}[1](M \times \mathfrak{g}[1] \times \mathfrak{g}[2])
$$

and the $B F V_{1}$-charge $\Theta$ is given in (5.23). Notice that $C(\mathcal{M})=\mathfrak{X}(M) \otimes W(\mathfrak{g}) \otimes C\left(\mathfrak{g}^{*}[0] \times\right.$ $\left.\mathfrak{g}^{*}[-1]\right)$. For the BFV differential $Q=\{\Theta$,$\} , we get in particular$

$$
\begin{aligned}
Q\left(\xi_{(1)}^{a}\right) & =\xi_{(0)}^{a}-\frac{1}{2} f_{b c}^{a} \xi_{(1)}^{b} \xi_{(1)}^{c} \\
Q\left(\xi_{(0)}^{a}\right) & =-f_{b c}^{a} \xi_{(1)}^{b} \xi_{(0)}^{c} \\
Q(\alpha) & =d_{\pi_{M}} \alpha+\xi_{(1)}^{a} L_{v_{a}} \alpha-\xi_{(0)}^{a} i_{d\left(\mu_{G}^{(0)}\right)_{a}} \alpha
\end{aligned}
$$

where $\alpha \in \mathfrak{X}(M)$. Thus $B:=\mathfrak{X}(M) \otimes W(\mathfrak{g})$ is a sub DGA of $(C(\mathcal{M}), Q)$ and let us denote by $d_{K}=\left.Q\right|_{B}$ the induced differential. We recognize the Weil differential $d_{W}$ as the restriction $\left.d_{K}\right|_{W(\mathfrak{g})}$. Moreover, the differential $d_{K}$ is analogous to the one defined by Kalkman ([22]) for his BRST model of equivariant de Rham cohomology.

We can thus follow 22] further to get an isomorphism $\psi: B \rightarrow A$ of DGA's defined by $\psi=\exp \left(-\xi_{(1)}^{a} i_{d\left(\mu_{G}^{(0)}\right)_{a}}\right)$ so that we get a chain of inclusions

$$
\left(A_{b}, d_{A_{b}}\right) \hookrightarrow\left(A, d_{A}\right) \simeq \psi\left(B, d_{K}\right) \hookrightarrow(C(\mathcal{M}), Q)
$$

Moreover, analogously to [22], $\psi^{-1}\left(A_{b}\right)=\left(\mathfrak{X}(M) \otimes S\left(\mathfrak{g}^{*}\right)\right)^{G}$ gives the Cartan model for Poisson equivariant cohomology ([16]). 
Finally, as observed in Subsection 5.1, the Poisson-Weil model of [35, 36] is the corresponding BFV-AKSZ model so that the above construction gives a clear explanation of the relation between one sector of the observables of the Poisson-Weil model with Poisson equivariant cohomology as observed in [36].

Remark 30 In the case where we deal with a general Poisson-Lie action with moment map $\mu_{G}^{(0)}: M \rightarrow G^{*}$, one would need to consider a non-linear version of the BF $V_{1}$ construction as in Section 5.1.

\section{E The zero modes case of AKSZ commuting with BFV}

In this section we give the details of the proof of the statement of Section 4.2 about AKSZ commuting with BFV in the context of finite dimensional 'zero modes' AKSZ theories (see details in [7]).

Let $X_{\Sigma}$ be the sheaf over a point corresponding to the cohomology ring $H_{d R}\left(\Sigma_{n+1}\right)$ of the source and $\left(M_{n}, \omega_{M_{n}}, \theta_{M_{n}}, C_{n}\right)$ a degree $n$ symplectic manifold endowed with reduction data $C_{n}$. Assume that $\Sigma_{n+1}$ is compact without boundary. The zero modes field space is

$$
\mathfrak{F}_{0}^{Z}=\operatorname{Map}\left\{X_{\Sigma} \rightarrow M_{n}\right\}
$$

and the evaluation map is denoted by $e v_{0}: \mathfrak{F}_{0}^{Z} \times X_{\Sigma} \rightarrow M_{n}$. From integration over $\Sigma_{n+1}$ we get an integration $\int d s$ defined by $\int d s s=1$ for $s \in H_{d R}^{n+1}\left(\Sigma_{n+1}\right)$ being a chosen normalized nontrivial top form. Using these ingredients, one can endow $\mathfrak{F}_{0}^{Z}$ with a degree -1 symplectic structure $\omega_{\mathfrak{F}_{0}^{Z}}$ via an analogous formula to the one on $\mathfrak{F}_{0}$ as described in Section 2.2. Choosing a basis $\alpha_{(l), i_{l}}$ for $H_{d R}^{l}\left(\Sigma_{n+1}\right)$, we get for any function on the target $f \in C\left(M_{n}\right)$

$$
e v_{0}^{*} f=\sum_{l, i_{l}} f^{(l), i_{l}} \alpha_{(l), i_{l}}
$$

where $f^{(l), i_{l}} \in C\left(\mathfrak{F}_{0}^{Z}\right)$. One can then show by direct computation the following:

Lemma 31 Assume $C_{n}$ is regular and given as a level set $C_{n}=\left\{\mu_{a}=0\right\} \subset M_{n}$, then

- The submanifold $\mathfrak{C}_{\mu}^{Z}:=\operatorname{Map}\left\{X_{\Sigma} \rightarrow C_{n}\right\} \subset \mathfrak{F}_{0}^{Z}$ is given by the vanishing of all components

$$
\mu_{a}^{(l), i_{l}}=0
$$

$$
\text { of } e v_{0}^{*} \mu_{a} \text {. }
$$


- $\mathfrak{C}_{\mu}^{Z}$ is coisotropic inside of $\mathfrak{F}_{0}^{Z}$, namely,

$$
\left\{\mu_{a}^{(l), i_{l}}, \mu_{b}^{(m), j_{m}}\right\}_{\mathfrak{F}_{0}^{Z}} \in I\left\langle\mu_{c}^{(k), i_{k}}\right\rangle
$$

- when $f \in C\left(M_{n}\right)$ is reducible by $C_{n}$, i.e. $\left\{\mu_{a}, f\right\}_{M_{n}}=A_{a}^{c} \mu_{c}$, then $f^{Z}:=\int d s$ ev $v_{0}^{*} f$ is reducible by $\mathfrak{C}_{\mu}^{Z}$ :

$$
\left\{\mu_{a}^{(l), i_{l}}, f^{Z}\right\}_{\mathfrak{F}_{0}^{Z}} \propto I\left\langle\mu_{c}^{(k), i_{k}}\right\rangle
$$

In particular, the zero modes $\mathrm{BV}$ action $S_{\theta}^{Z}=\int d s e v_{0}^{*} \theta_{M_{n}} \in \mathfrak{F}_{0}^{Z}$ is reducible by $\mathfrak{C}_{\mu}^{Z} \subset \mathfrak{F}_{0}^{Z}$ since $\theta_{M_{n}}$ is reducible by $C_{n} \subset M_{n}$.

We thus get that $\left(\mathfrak{F}_{0}^{Z}, \omega_{\mathfrak{F}_{0}^{Z}}, S_{\theta}^{Z}, \mathfrak{C}_{\mu}^{Z}\right)$ defines reduction data on $\mathfrak{F}_{0}^{Z}$. A $B F V_{-1}$ model for this reduction data will be thus given by a degree -1 symplectic manifold that looks like

$$
B F V_{-1} \approx \mathfrak{F}_{0}^{Z} \times\left\{\eta_{(l), i_{l}}^{a}\right\} \times\left\{p_{\eta^{a}}^{(l), i_{l}}\right\}
$$

where we have one ghost $\eta_{(l), i_{l}}^{a}$ for each constraint $\mu_{a}^{(l), i_{l}} \in C\left(\mathfrak{F}_{0}^{Z}\right)$ while the $p_{\eta^{a}}^{(l), i_{l}}$ denote their conjugate antighosts. The corresponding $B F V_{-1}$ charge will take the form

$$
\Theta^{Z}:=\sum_{l, i_{l}} \mu_{a}^{(l), i_{l}} \eta_{(l), i_{l}}^{a}+S_{\theta}^{Z}+O\left(p_{\eta}\right)
$$

Notice that $\Theta^{Z}$ must have degree 0 as $S_{\theta}^{Z}$ does and that, then, the degree of the ghosts $\eta_{(l), i_{l}}^{a}$ must be $\left(l-k_{a}\right)$ where $k_{a}$ is the degree of $\mu_{a} \in C\left(M_{n}\right)$.

With this set up, we can then state that AKSZ commutes with BFV:

Proposition $32 \operatorname{Let}(\mathcal{M}, \Omega, \Theta)$ be a $B F V_{n}$ model for the target reduction data $\left(M_{n}, \omega_{n}, \theta_{n}, C_{n}\right)$. Then, the construction $\left(\mathfrak{F}^{Z}, \omega_{\mathfrak{F}^{Z}}, \Theta^{Z}\right):=A K S Z\left(X_{\Sigma},(\mathcal{M}, \Omega, \Theta)\right)$ gives a BFV $V_{-1}$ model for the reduction data $\left(\mathfrak{F}_{0}^{Z}, \omega_{\mathfrak{F}_{0}^{Z}}, S_{\theta}^{Z}, \mathfrak{C}_{\mu}^{Z}\right)$.

Proof: Let us consider the identification

$$
\eta_{(l)}^{a}=\xi^{a,(m)}
$$

for $m=n+1-l$, with $e v_{0}^{*} \xi^{a}=\sum_{m, i_{m}} \xi^{a,(m), i_{m}} \alpha_{(m), i_{m}}$ and $\xi^{a}$ being the ghost in $\mathcal{M}$ associated to the constraint $\mu_{a}$ on the target $M_{n}$. Then, one can direclty check that by applying the AKSZ construction, both the space $\mathfrak{F}^{Z}=\operatorname{Map}\left\{X_{\Sigma} \rightarrow \mathcal{M}\right\}$ and the charge $\Theta^{Z}=\int d s e v_{0}^{*} \Theta$ have the desired form (E.41) and (E.42), respectively. Moreover, by construction, $\Theta^{Z}$ is homological, $\left\{\Theta^{Z}, \Theta^{Z}\right\}=0$. 
Remark 33 Recall from [7], that the space of zero modes $\mathfrak{F}_{0}^{Z}$ can be obtained from the full $A K S Z$ field space $\mathfrak{F}_{0}=\left\{T[1] \Sigma_{n+1} \rightarrow M_{n}\right\}$ via reduction $\mathfrak{F}_{0}^{Z}=\mathfrak{F}_{0} / / \mathfrak{Z}$ by the coisotropic $\mathfrak{Z}=\left\{\Phi \in \mathfrak{F}_{0}: D \Phi=0\right\}$. One can check that reduction by $\mathfrak{Z}$ is formaly compatible with reduction by $\mathfrak{C}_{0}:=\left\{T[1] \Sigma_{n+1} \rightarrow C_{n}\right\}$. In fact, one can also obtain the computations of Lemma 31 directly as reduction by $\mathfrak{C}_{0}$ of formal computations on $\mathfrak{Z} \subset \mathfrak{F}_{0}$. In particular, imposing $D \Phi=0$ brings reduction data $\left(\mathfrak{F}_{0}, \omega_{\mathfrak{F}_{0}}, S_{\theta}, \mathfrak{C}_{0}\right)$ to the zero modes reduction data $\left(\mathfrak{F}_{0}^{Z}, \omega_{\mathfrak{F}_{0}^{Z}}, S_{\theta}^{Z}, \mathfrak{C}_{\mu}^{Z}\right)$.

\section{References}

[1] M. Alexandrov, M. Kontsevich, A. Schwartz and O. Zaboronsky, "The Geometry of the master equation and topological quantum field theory," Int. J. Mod. Phys. A 12 (1997) 1405 [arXiv:hep-th/9502010].

[2] I. Batalin, E. Fradkin, "A generalized canonical formalism and quantization of reducible gauge theories", Phys. Lett. 122B (1983), 157.

[3] I. Batalin and G Vilkovisky, "Gauge algebra and quantization", Phys. Lett., 102B (1981) 27.

[4] I. Batalin, and G. Vilkovisky, "Quantization of gauge theories with linearly dependent generators", Phys. Rev. D29 (1983), 2567.

[5] F. Bonechi, A. S. Cattaneo and P. Mnëv, "The Poisson sigma model on closed surfaces," J.High Energy Phys. 2012 JHEP01(2012)099 [arXiv:1110.4850 [hep-th]].

[6] F. Bonechi and M. Zabzine, "Poisson sigma model on the sphere", Commun. Math. Phys. 285 (2009) 1033 [arXiv:hep-th/0706.3164].

[7] F. Bonechi, P. Mnëv and M. Zabzine, "Finite dimensional AKSZ-BV theories," Lett. Math. Phys. 94 (2010) 197 [arXiv:0903.0995 [hep-th]].

[8] H. Bursztyn, M. Crainic and P. Severa, "Quasi-Poisson structures as Dirac structures," Travaux Mathématiques XVI (2005), 41-52.

[9] H. Bursztyn, G. R. Cavalcanti and M. Gualtieri "Reduction of Courant algebroids and generalized complex structures," Adv. Math., 211 (2), 2007, 726-765 [math/0509640[math.DG]]

[10] H. Bursztyn, A. Cattaneo, R. Mehta and M. Zambon, in preparation. 
[11] A. Cabrera and H.-C. Herbig, "BFV complex for graded manifolds," in preparation.

[12] A. Cattaneo and G. Felder, "On the AKSZ formulation of the Poisson Sigma Model". Lett.Math.Phys. 562 (2001) 163-179. [arXiv:math/0102108]

[13] A. Cattaneo, P. Mnev, N. Reshetikhin, "Classical BV theories on manifolds with boundaries", [arXiv:math-ph/1201.0290]

[14] A. Cattaneo and M.Zambon, "A supergeometric approach to Poisson reduction", [arxiv:math/1009.0948].

[15] L. D. Faddeev, "Feynman integral for singular Lagrangians," Theor. Math. Phys. 1, 1 (1969) [Teor. Mat. Fiz. 1, 3 (1969)].

[16] V. L. Ginzburg, "Equivariant Poisson cohomology and a spectral sequence associated with a moment map". Internat. J. Math., 10 (1999), 977-1010 [arxiv:dg-ga/9611002].

[17] V.L.Ginzburg and A.Weinstein, "Lie Poisson structure on some Poisson Lie groups", J. Amer. Math. Soc. 5 (1992), 445.

[18] M. Grigoriev and P.H. Damgaard, "Superfield BRST Charge and the Master Action," Phys.Lett. B474 (2000) 323-330 [arXiv:hep-th/9911092].

[19] M. Henneaux, "Hamiltonian form of the path integral for theories with a gauge freedom", Physics Reports, 126, 1,(1985) 1-66.

[20] M. Henneaux and C. Teitelboim, Quantization of gauge systems. Princeton University Press, Princeton, (1992).

[21] H-C. Herbig: "Variations on Homological Reduction", PhD thesis [arXiv:0708.3598[math.QA]].

[22] J. Kalkman, A BRST model applied to symplectic geometry, PhD Thesis Utrecht, 1993 [hep-th/9308132].

[23] T. Kimura, "Generalized classical BRST and reduction of Poisson manifolds". Comm Math Phys 151 (1993) 155-182.

[24] Z.-J. Liu, A. Weinstein and P. Xu, "Manin triples for Lie bialgebroids", J. Diff. Geom. 45 (1997), 547-574 [arxiv:dg-ga/9508013].

[25] A. Losev, "BV formalism and quantum homotopical structures," Lectures at GAP3, Perugia, 2005. 
[26] H-J. Lu, Multiplicative and Affine Poisson Structures on Lie Groups, PhD thesis, Berkeley, can be downloaded from http://hkumath.hku.hk/ jhlu/thesis.pdf

[27] S.L. Lyakhovich and A.A. Sharapov, "BRST theory without Hamiltonian and Lagrangian", J.High Energy Phys. 2005, JHEP03 (2005) [arXiv:hep-th/0411247]

[28] P. Mnëv, "Discrete BF theory", PhD thesis, [arXiv:08091160].

[29] J. Qiu and M. Zabzine, "Introduction to Graded Geometry, Batalin-Vilkovisky Formalism and their Applications," Archivum Math. 47, 143 (2011) [arXiv:1105.2680 [math.QA]].

[30] D. Roytenberg, "AKSZ-BV formalism and Courant algebroid-induced topological field theories," Lett. Math. Phys. 79 (2007) 143 [arXiv:hep-th/0608150].

[31] F. Schätz, "Invariance of the BFV-complex," Pacific J. Math. 248 (2010), no. 2, 453-474 [arXiv:math/0812.2357]

[32] A. S. Schwarz, "Geometry of Batalin-Vilkovisky quantization," Commun. Math. Phys. 155, 249 (1993) [hep-th/9205088].

[33] D. Signori, Sottovarieta coisotrope in teoria di campo e quantizzazione, Laurea thesis, Milan University, can be downloaded from http://user.math.uzh.ch/cattaneo/signori.pdf

[34] J. D. Stasheff, "Homological reduction of constrained Poisson algebras", J. Diff. Geom. 45 (1997) 221-240 [q-alg/9603021].

[35] R. Zucchini: "The Hitchin Model, Poisson-quasi-Nijenhuis Geometry and Symmetry Reduction”, J.High Energy Phys. 2007 JHEP10(2007)075 [arXiv:0706.1289[hep-th]]

[36] R. Zucchini: "Gauging the Poisson sigma model", J.High Energy Phys. 2008 JHEP05(2008) [arXiv:0801.0655[hep-th]]

[37] R. Zucchini, "The gauging of BV algebras", J.Geom.and Phys.60 11 (2010), 18601880 [arXiv:1001.0219[hep-th]] 